\title{
Merger induced clump formation in distant infrared luminous starburst galaxies
}

\author{
Antonello Calabrò ${ }^{1}$, Emanuele Daddi ${ }^{1}$, Jérémy Fensch ${ }^{2,3}$, Frédéric Bournaud ${ }^{1}$, Anna Cibinel ${ }^{4}$, Annagrazia Puglisi ${ }^{1}$, \\ Shuowen $\mathrm{Jin}^{5,6}$, Ivan Delvecchio ${ }^{1}$, and Chiara D'Eugenio ${ }^{1}$

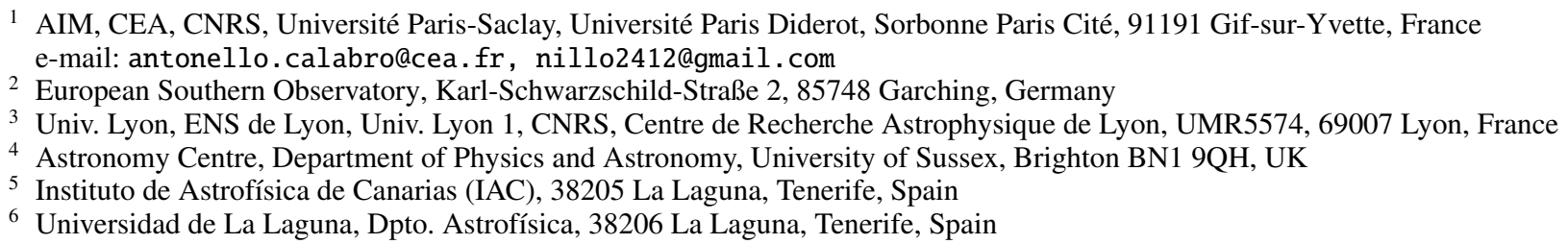

Received 26 April 2019 / Accepted 2 October 2019

\begin{abstract}
While the formation of stellar clumps in distant galaxies is usually attributed to gravitational violent disk instabilities, we show here that major mergers also represent a competitive mechanism to form bright clumps. Using $\sim 0.1^{\prime \prime}$ resolution ACS F814W images in the entire COSMOS field, we measured the fraction of clumpy emission in 109 main sequence (MS) and 79 Herschel-detected starbursts (off-MS) galaxies at $0.5<z<0.9$, representative of normal versus merger induced star-forming activity, respectively. We additionally identify merger samples from visual inspection and from Gini-M20 morphological parameters. Regardless of the merger criteria adopted, the clumpiness distribution of merging systems is different from that of normal isolated disks at a $>99.5 \%$ confidence level. The former reaches higher clumpiness values up to $20 \%$ of the total galaxy emission. We confirm the merger induced clumpiness enhancement with novel hydrodynamical simulations of colliding galaxies with gas fractions typical of $z \sim 0.7$. Multi-wavelength images of three starbursts in the CANDELS field support the young nature of clumps, which are likely merger products rather than older preexisting structures. Finally, for a subset of 19 starbursts with existing near-infrared rest frame spectroscopy, we find that the clumpiness is mildly anti-correlated with the merger phase, which decreases toward final coalescence. Our result can explain recent ALMA detections of clumps in hyperluminous high- $z$ starbursts, while normal objects are smooth. This work raises a question as to the role of mergers on the origin of clumps in high redshift galaxies in general.
\end{abstract}

Key words. galaxies: evolution - galaxies: interactions - galaxies: high-redshift - galaxies: structure - galaxies: star formation galaxies: starburst

\section{Introduction}

While local galaxies have well defined morphological types described by the so called Hubble sequence, higher redshift systems are more irregular and clumpy, which makes it increasingly more difficult to associate one of the Hubble classes to them. At redshift $\sim 0.6$, for example, the fraction of irregular systems increases by $40 \%$ compared to the local Universe (Delgado-Serrano et al. 2010). The clumpy substructures detected in the average star-forming galaxy population at intermediate and high redshift represent aggregations of relatively young stars (depending on the observed spectral window), which arise from a smoother and fainter disk luminosity profile. They have stellar masses ranging $10^{7}-10^{9} M_{\odot}$ and sizes of approximately $100-1000 \mathrm{pc}$ (Elmegreen et al. 2005), and are typically identified through highly resolved observations in the restframe ultraviolet (UV; e.g., Chapman et al. 2003; Puech 2010), optical (e.g., Murata et al. 2014), and near-infrared (near-IR; Förster Schreiber et al. 2006, 2009).

Clumpy galaxies at redshift $>0.5$ with stellar masses of $10^{10}-10^{11} M_{\odot}$ are thought to be the progenitors of presentday spirals (Elmegreen et al. 2005, 2013; Bournaud et al. 2007;
Ceverino et al. 2010). Moreover, typical clumps at $z>1 \mathrm{might}$ live between 100 and $650 \mathrm{Myr}$, depending on their stellar mass (Zanella et al. 2015, 2019). As a consequence, they could migrate toward the center and eventually contribute to the formation of a central bar (Immeli et al. 2004a; Sheth et al. 2012; Kraljic et al. 2012) or to the stellar bulge growth (Noguchi 1999; Elmegreen et al. 2008; Bournaud et al. 2014; Bournaud 2016). This contributes to stabilize the disks (e.g., Ceverino et al. 2010) and to determine the final imprint for the morphological shapes encoded in the Hubble sequence. According to Kraljic et al. (2012), local Milky way-like spirals acquire their disk morphology at $z \sim 0.8-1$, and they completely stabilize at redshift 0.5 or lower (Cacciato et al. 2012). Alternatively, clumps in distant galaxies may lead to the formation of super star clusters and globular clusters (Shapiro et al. 2010).

The formation and origin of clumps at all redshifts is not completely assessed as it can be ascribed to different triggering mechanisms. Usually, in clumpy galaxies at $z>1$, they are thought to be triggered by violent disk instabilities in highly gasrich, dense, and turbulent disks. Additionally, they are continuously fed by cold gas streams from the circumgalactic medium (CGM) and the cosmic web (Dekel et al. 2009). These can 
sustain the high gas fractions $f_{\text {gas }}$ on the order of $\sim 0.4-0.5$ that are typically found in high redshift galaxies (Daddi et al. 2010; Tacconi et al. 2010; Rodrigues et al. 2012).

Below $z \sim 1$, cosmological simulations predict a strong cutoff of cold flow accretion into galaxies (Kereš et al. 2005; Dekel \& Birnboim 2006), which might indirectly result in the decrease of the average star formation rate (SFR) density in the Universe (Madau \& Dickinson 2014) and of the gas content in galaxies down to $f_{\text {gas }} \sim 0.2-0.4$. This strong suppression of gas fractions may then affect the physical properties and abundance of clumps, and could require alternative mechanisms of formation and evolution. For example, smooth accretion of gas from tidally disrupted companions or stripped satellites in cluster environments are suggested to feed some low-mass, local analogs of high- $z$ clumpy galaxies (Garland et al. 2015). Instead, a more relevant additional channel for producing clumps at any host stellar mass and epoch is represented by major mergers, as proposed in Somerville (2001), Lotz et al. (2004), Di Matteo et al. (2008). However, at intermediate redshifts, there is no general consensus yet as to the role and importance of mergers for the formation of clumps. Additionally, while mergers produce very dense and compact starbursting cores, it is still unclear as to what fraction of star formation occurs in off-nuclear regions, possibly in the form of clumps.

From an observational point of view, Puech (2010) analyzed UV rest-frame clumps in 11 clumpy galaxies at $z \sim 0.6$. He claims that interactions may be the dominant driver for the formation of clumps at that epoch because of the complex kinematic structure observed for half of them. In contrast, Guo et al. (2015) and Murata et al. (2014) argue that major mergers have a negligible role explaining the fraction of clumpy galaxies at all masses at $z \lesssim 1.5$, suggesting instead a prevalent role of violent disk instabilities or minor mergers. Similarly, Bournaud et al. (2012) found that clumpy low-mass star-forming galaxies at $z \sim 0.7$ are comparable to gas-rich turbulent disks observed at higher redshifts, suggesting that gravitational instabilities are the most important cause of gas fragmentation and the formation of clumps.

However, even with different sample selections and observed photometric bands, these studies are only focused on the main origin of clumpy galaxies among the whole population. Since they do not perform a merger identification, they neither analyze the implications that mergers have on clumps formation, nor they quantitatively measure the clumpiness parameter (i.e., fraction of light in clumps) in comparison to normal disks. Puech (2010) show a possible connection between mergers and UV clumps, but many of their substructures are no longer detected in the optical, thus they cannot be directly compared with our sample. Moreover, their subset is not representative of the whole merger population at intermediate redshifts, given the strict requirements of their selection criteria.

The connection between mergers and clumps is not clear also in hydrodynamical simulations: while some studies suggest that merger events can trigger turbulent modes in the ISM that lead to rapid fragmentation of gas and formation of clumps (Teyssier et al. 2010; Bournaud et al. 2011a; Renaud et al. 2014), other morphological studies on simulated galaxies do not see at all an enhancement of clumpy emission during the merger (e.g., Nevin et al. 2019). The reason of these discrepancies resides in the different resolutions adopted, in the modeling of feedback and turbulence, and in the specific initial conditions considered, such as the merger geometry, the stellar mass ratio, and the initial gas fraction $f_{\text {gas }}$ of colliding galaxies (e.g., Di Matteo et al. 2008; Governato et al. 2010; Fensch et al.
2017). Most of the simulations focus on local or high redshift galaxies, thus they have lower or higher gas fractions than the typical values at intermediate redshifts. The few simulations with an intermediate $f_{\text {gas }}$ value (Cox et al. 2006; Di Matteo et al. 2008) did not allow the gas to cool down below $10^{4} \mathrm{~K}$, which is necessary to study the evolution of the gas structure during the interactions (Teyssier et al. 2010; Bournaud et al. 2011b). In other cases, the resolutions are too low for the sub-kpc spatial scales that we want to investigate to detect clumpy structures (Sparre \& Springel 2016; Rodriguez-Gomez et al. 2016). Given all these uncertainties, more observations and constraints are needed to clarify whether mergers can trigger the formation of clumps at intermediate redshift, and to put constraints on feedback models in merger simulations.

In order to test this connection with observations, multiple merger identification criteria are applicable. In the local Universe, for example, a deep connection has been established between mergers and ultra-luminous infrared galaxies (ULIRGs; Sanders \& Mirabel 1996; Clements et al. 1996), which are highly obscured systems with total infrared luminosities exceeding $10^{12} \mathrm{~L}_{\text {sun }}$, which reflects a high and obscured star-formation activity. At higher redshifts, dusty starburst (SB) galaxies, defined by their SFRs much higher compared to normal star-forming systems on the main sequence (MS; Noeske et al. 2007; Daddi et al. 2007; Elbaz et al. 2007), could be the analogs of local ULIRGs. However, their nature is still hotly debated: some studies claim they may be gas rich galaxies undergoing anomalous gas accretion events (e.g., Scoville et al. 2016), while other works show that the most extreme cases are mergers, displaying disturbed morphologies (Elbaz \& Cesarsky 2003). According to the latter scenario, starbursts and main sequence galaxies are associated, respectively, to a highly efficient starformation mode and gas consumption induced by major merger events, and to a quasi-steady star-forming activity with much longer gas depletion timescales on the order of $\sim 1-2 \mathrm{Gyr}$ (Sargent et al. 2014; Silverman et al. 2015). Building on this previous knowledge, Calabrò et al. (2018) find that starbursts at $0.5<z<0.9$ are mostly merger triggered, as revealed either by their morphology or by the presence of extremely obscured starbursting cores. In addition, Cibinel et al. (2019) show that the merger fraction above the main sequence is of approximately $80 \%$. Even though the longstanding debate has not yet concluded, these latter studies give a strong motivation for searching ongoing merging systems among the starburst galaxy population. This approach would be complementary to the visual inspection and to non parametric merger estimators (Conselice et al. 2003; Lotz et al. 2004), which could be highly incomplete and very difficult to perform for distant objects. In addition, it could be also more reliable compared to the kinematics: Law et al. (2009) show indeed that clear merger candidates at $z>1$ can have regular rotation patterns typical of disk galaxies.

The COSMOS field allows to select so far the largest statistical sample of starbursts with plenty of ancillary data, including an almost complete imaging coverage in at least one HST filter (F814W, i.e., approximately $i$-band), photometric data ranging from UV to far-IR and sub-mm (Laigle et al. 2016; Jin et al. 2018), and infrared based SFRs (Jin et al. 2018). In this paper, we exploit high resolution $\left(0.095^{\prime \prime}\right)$ HST/ACS F814W public images over the whole $\simeq 2 \mathrm{deg}^{2}$ COSMOS field to build a large sample of starburst galaxies and investigate with unprecedented statistics the role of mergers on the formation of clumps at intermediate redshifts. To this aim, we compare the starbursts to a control sample of randomly selected isolated main 
sequence galaxies at the same cosmic epoch. At these redshifts, $i$-band images probe the optical rest-frame spectral range, which, compared to UV emission (dominated by young massive O-B stars), is more sensitive to the light of intermediate age (A to $\mathrm{G}$ type) stars, and thus it is more a tracer of the stellar mass than of the ongoing SFR. In addition, these observations are less affected by dust attenuation compared to UV rest-frame images of those clumpy galaxies studied systematically at high redshift $(z \gg 1)$.

We also present a set of numerical simulations of merger interactions between typical $z \sim 0.7$ galaxies, which could help to interpret our observational results. To this aim, these simulations consider the correct gas fractions for this cosmic epoch $\left(f_{\text {gas }} \simeq 0.3\right)$, while simultaneously allowing the gas component to cool below $10^{4} \mathrm{~K}$ and to be resolved on small spatial scales of $6 \mathrm{pc}$, necessary to study its evolution during the merger.

The paper is organized as follows. In Sect. 2 we describe our selection of starburst galaxies and of a control sample of main sequence systems in COSMOS field. We also present their HST F814W images, from which we derive basic morphological properties and an estimation of the clumpiness parameter. After showing our observational results in Sect. 3, we describe in Sect. 4 the hydrodynamical simulations used to verify the connection between mergers and clumps at intermediate redshift. Then we discuss possible physical interpretations of our findings, and we show the summary and conclusions in Sect. 5. We adopt the Chabrier (2003) initial mass function, $\mathrm{AB}$ magnitudes, and standard cosmology $\left(H_{0}=70 \mathrm{~km} \mathrm{~s}^{-1} \mathrm{Mpc}^{-1}, \Omega_{\mathrm{m}}=0.3\right.$, $\left.\Omega_{\Lambda}=0.7\right)$.

\section{Methodology}

In this section we describe our sample selection and the derivation of the morphological properties from single-band HST images, also quantifying, through the clumpiness parameter, the contribution of off-nuclear clumps to the galaxy emission.

\subsection{Starbursts and main sequence galaxies selection}

As mentioned in the Introduction, we argued in a previous work (Calabrò et al. 2018) that infrared-luminous starbursts at $0.5<$ $z<0.9$ are mostly major mergers. For this reason, focusing on the same redshift range, we decided to analyze all starbursts in the full COSMOS field to test the effect of mergers on the formation and evolution of clumps, while using main sequence isolated galaxies as a control sample.

All the galaxies in this work were selected from the IR+(sub)mm catalog of Jin et al. (2018), which includes $\sim 15 \mathrm{k}$ star-forming galaxies in COSMOS, with deblended photometry ranging from Spitzer $24 \mu \mathrm{m}$ to VLA $1.4 \mathrm{GHz}$ bands. The stellar masses $\left(M_{*}\right)$ of the sources are computed by Laigle et al. (2016) at the photometric redshifts by fitting 16 bands from nearultraviolet to mid-infrared. As explained in Calabrò et al. (2018), using the spectroscopic redshift (when available) does not affect significantly the stellar mass derivations, as they are in general agreement within the uncertainties of 0.1 dex reported by the same authors.

We adopted the infrared SFRs (=SFR $\mathrm{IR}$ ) derived by Jin et al. (2018) by fitting multi-wavelength broad-band photometric data from IRAC to radio VLA bands. Four components were used to determine the best-fit SED: a Bruzual \& Charlot (2003) stellar model (at different ages and metallicities, constant star-formation history (SFH), Chabrier IMF and Calzetti et al.
2000 attenuation law), a mid-infrared template for AGNs (Mullaney et al. 2011), and Draine \& Li (2007) dust emission models. When available, the spectroscopic redshift was fixed in the fit. For main sequence galaxies that are undetected in the $100 \mu \mathrm{m}-850 \mu \mathrm{m}$ bands (including Herschel and SCUBA2 photometry) at signal-to-noise ratio $S / N_{\text {IR }}<5$, the SFRs were computed from their $24 \mu \mathrm{m}$ fluxes as explained in Jin et al. (2018). Finally, we calculated the total SFRs as

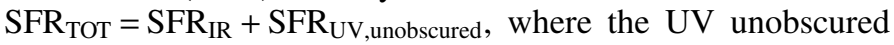
SFRs were inferred from their Laigle et al. (2016) $u$-band total magnitudes, as shown in Calabrò et al. (2018).

Following the procedure described in Calabrò et al. (2018, 2019), we compared the $M_{\star}$ and SFR of our galaxies to the starforming main-sequence of Schreiber et al. (2015) (which was shown to agree well with our data) in order to derive the distance from the main sequence $\operatorname{dist}_{\mathrm{MS}}=\log _{10}\left(\mathrm{SFR} / \mathrm{SFR}_{\mathrm{MS}}\right)$. Finally, starbursts are taken above the typical threshold of dist $_{\mathrm{MS}}>0.6$ (i.e., above a factor of four), following Rodighiero et al. (2011).

We required also the redshift, either spectroscopic or photometric (from Jin et al. 2018), to be in the range 0.5-0.9. As mentioned in the Introduction, we want to focus on the intermediate redshift regime to build-up on the growing understanding generated by our previous works (Calabrò et al. 2018, 2019). This would allow the follow-up of some of the clumps with nearinfrared integral-field spectrographs to derive $\mathrm{H} \alpha$ and $\mathrm{Pa} \beta$ emission line maps. In addition, it is in this cosmic epoch that we may have the final imprint on the morphology of present-day galaxies.

As an additional constraint for our sample, we imposed the stellar mass $M_{*}$ to be greater than $10^{10} M_{\odot}$, since above this limit and up to $z=0.9$, all SBs would be Herschel-detected at $S / N_{\text {IR }}>5$ (see Fig. 13 in Jin et al. 2018), and we have a masscomplete sample of normal star-forming galaxies down to a factor of three below the main sequence.

These three criteria yielded a subset of 118 Herscheldetected $\left(S / N_{\text {IR }}\right)$ starbursts, from which we discarded two residual quasar-like objects and additional 20 galaxies without HST-ACS coverage, leaving a sample of 96 starbursts in total.

We then selected a control sample of $145 \mathrm{MS}$ galaxies with HST F814W images, in order to have a larger statistics and avoid that additional sample cuts in our analysis would produce a lower number of MS systems than SBs. Our normal star-forming galaxies were selected with a random generator within $\Delta$ dist $_{M S}$ of $\pm 0.47 \mathrm{dex}$ (a factor of three) from the MS, in the same redshift and stellar mass range defined for SBs, and requiring that they are star-forming according to the NUV-R/R-J diagram to avoid quiescent galaxies (Laigle et al. 2016).

Even though this latter subset is representative of secularly evolving star-forming disks, we remember that it may contain also a fraction of ongoing mergers. Indeed, at our redshifts, the merger fraction is expected to be higher than the relative number of starbursts (Schreiber et al. 2015), both because of SFR fluctuations during the merger process itself (Di Matteo et al. 2008) and because it might be more difficult for mergers to trigger starbursts as in the local Universe (Fensch et al. 2017). We discuss several additional methods to identify mergers in Sect. 2.3.

\subsection{HST images and morphology}

In order to study the morphology and the presence of clumpy structures in our galaxies, we need high resolution images that can probe spatial scales significantly below $1 \mathrm{kpc}$. For the sample analyzed in this paper, we adopted F814W ACS images (Koekemoer et al. 2007), which we retrieved from the COSMOS 
web service ${ }^{1}$. These represent so far the deepest and highest resolution publicly available data in this field, with $\sim 2000 \mathrm{~s}$ time integration, a magnitude limit of $25.61 \mathrm{mag}$ for extended sources (assuming a circular aperture radius of $0.3^{\prime \prime}$ ) and a median FWHM resolution of $\sim 0.095^{\prime \prime}$ (with a pixel scale of $0.03^{\prime \prime}$ pixel $^{-1}$ ). At our redshifts, this means that we are able to distinguish substructures with separations of at least $\simeq 600 \mathrm{pc}$ at $z=0.5$ to $\simeq 800 \mathrm{pc}$ at $z=0.9$, and thus detect clumps with FWHM sizes on this scale, which is appropriate given those typically found in high-redshift clumpy galaxies. A representative subset of HST cutouts for the starburst and MS populations are displayed in Fig. 1 and 2.

In the first step of our analysis, we created a segmentation map of the HST F814W $\left(15^{\prime \prime} \times 15^{\prime \prime}\right)$ cutouts using the python package PHOTUTILS ${ }^{2}$. In brief, the code identifies the sources as groups of connected pixels with a flux higher than a threshold. The latter value is calculated at a given $\mathrm{S} / \mathrm{N}$ per pixel above the background, which is estimated from the entire cutout using a sigma-clipped statistics. We found that a $\mathrm{S} / \mathrm{N}$ threshold of 1.3 works well in all the cases, including the low surface-brightness external regions and wings, while separating different galaxies in the same cutout region. The few cases where two close-in-sky but spatially unrelated galaxies (i.e., located at completely different redshifts) were selected as the same source in the segmentation map, we applied the deblending function inside PHOTUTILS, keeping only the central object. The location of the final source was saved into a mask $M_{0}$ : we assigned a value of 1 to all the pixels inside the galaxy contours identified by the segmentation map, and 0 to the others.

Afterward, we ran the galaxy morphology tool STATMORPH (Rodriguez-Gomez et al. 2019) to derive the elongation $\mathrm{E}$ of the galaxy contained in the previously selected region, which we expect can crucially affect the detectability of clumpy structures. $E$ is defined as $\frac{A}{B}$, where $A$ and $B$ are, as in SExtractor, the maximum and minimum rms dispersion of the object profile along all directions. Equivalently, they can be considered as the semi-major and semi-minor axis lengths of the ellipse that best describes the galaxy shape.

\subsection{Morphological merger classification}

In addition to the elongation, it is essential to better characterize the morphological properties to identify which galaxies in the main sequence could be possibly mergers. To this aim, we pursued two approaches: one relying on non-parametric quantitative estimators, and the second based on a visual analysis.

In the first approach, the Gini and $M_{20}$ coefficients, defined by Lotz et al. (2004), are usually adopted for selecting possible mergers and interacting systems. Gini $(G)$ measures the degree of inequality of the flux distribution among the pixels in the image, and is higher for galaxies with bright clumps and nuclei. For our HST cutouts, we computed G with the following formula:

$$
G=\frac{1}{\bar{X} n(n-1)} \sum_{i}^{n}(2 i-n-1) X_{i},
$$

where $n$ is the number of pixels of the galaxy (defined by the mask $\left.M_{0}\right), X_{i}$ are the counts in each pixel $i$ sorted in increasing order and $\bar{X}$ is the mean pixel value (Glasser 1962).

On the other hand, $M_{20}$ is defined as the normalized second order moment of the brightest $20 \%$ pixels of the galaxy:

\footnotetext{
1 http://irsa.ipac.caltech.edu/data/Cosmos/

2 https://doi.org/10.5281/zenodo. 2533376
}

$$
\begin{aligned}
& M_{20}=\log _{10}\left(\frac{\sum_{i} M_{i}}{M_{\mathrm{tot}}}\right), \text { with } \sum_{i} f_{i}<0.2 f_{\mathrm{tot}} \\
& \text { with } M_{\mathrm{tot}}=\sum_{i}^{n} M_{i}=\sum_{i}^{n} f_{i}\left[\left(x_{i}-x_{\mathrm{c}}\right)^{2}+\left(y_{i}-y_{\mathrm{c}}\right)^{2}\right] .
\end{aligned}
$$

In the above formula, $x_{i}$ and $y_{i}$ are the pixel coordinates, and $x_{\mathrm{c}}$ and $y_{\mathrm{c}}$ represent the galaxy's center, such that $M_{\mathrm{tot}}$ is minimized. $f_{i}$ are the counts in each pixel, while $f_{\text {tot }}$ symbolizes the total counts among galaxy pixels identified by the segmentation map. In short, this quantity measures the relative concentration of light around the position that minimizes $M_{20}$ itself. It is higher in the presence of bright bars, spiral arms, tidal tails, off-center clumps, and it is very sensitive, for example, to multiple nuclei. Both parameters were calculated with python routines by applying the Eqs. (1) and (2).

The typical uncertainties of Gini and $M_{20}$ estimations are of 0.02 and 0.1 , respectively. They were estimated by Lotz et al. (2008) from ACS F814W galaxy images at our same redshifts in the EGS field, at a depth comparable with our analysis $(\sim 2000 \mathrm{~s}$ of integration). In addition, the ULIRGs in their sample, to which they apply the classification, have $I_{\mathrm{F} 814 \mathrm{~W}}<23 \mathrm{mag}$, thus largely applicable to our case.

Since Gini and $M_{20}$ increase with the strength of merger signatures, we can use both to identify a subset of galaxies with merger morphologies. Following the classification criteria of Lotz et al. (2008), we defined a "mergerness" parameter $m$ as:

mergerness $=$ Gini $+0.14 \times M_{20}-0.33$,

where the coefficients were calibrated by Lotz et al. (2008) and do not vary with redshift (up to $z \simeq 1.2$ ). This quantity represents the difference between the Gini estimated and that required to classify the system as a merger. Therefore, according to this criterion, galaxies with $m>0$ are identified as morphological mergers throughout the paper. The exact mergerness values of all the galaxies are listed in Table A.1.

We remark that for our sample we cannot apply the morphological analysis performed by Cibinel et al. (2019) on resolved stellar mass maps, since it requires multi-wavelength images. However, our single band optical rest-frame images are more sensitive to the stellar mass of the system compared to UV restframe observations, and this dataset represents so far the best compromise if we need high spatial resolution information.

We show in Fig. 3-top the distribution of the Gini and $M_{20}$ coefficients for our galaxies, and highlight with the black continuous line the merger threshold of Lotz et al. (2008). In the bottom panel of Fig. 3 we display instead the histogram of mergerness parameters, separately for main sequence and starburst galaxies. These two populations have overall a different distribution of $m$ and different medians, where starbursts tend to have a higher mergerness compared to normal star-forming galaxies.

We notice that the threshold criterion of Lotz et al. (2008) defines a mergerness parameter space where SBs start to dominate in number over the MS population. In our case, $76 \%$ of the morphological merger systems $(m>0)$ turn out to be starbursts. However, this subset is not complete, as it represents a minor fraction $(43 \%)$ of all the starburst population in our sample. This result should not be surprising. Indeed, the observability timescale of a merger in the upper part of the Gini- $M_{20}$ diagram may not coincide with the starburst phase duration and depends on many factors, including the mass ratio and the gas fraction of colliding galaxies, the viewing angle, the impact geometry, the 


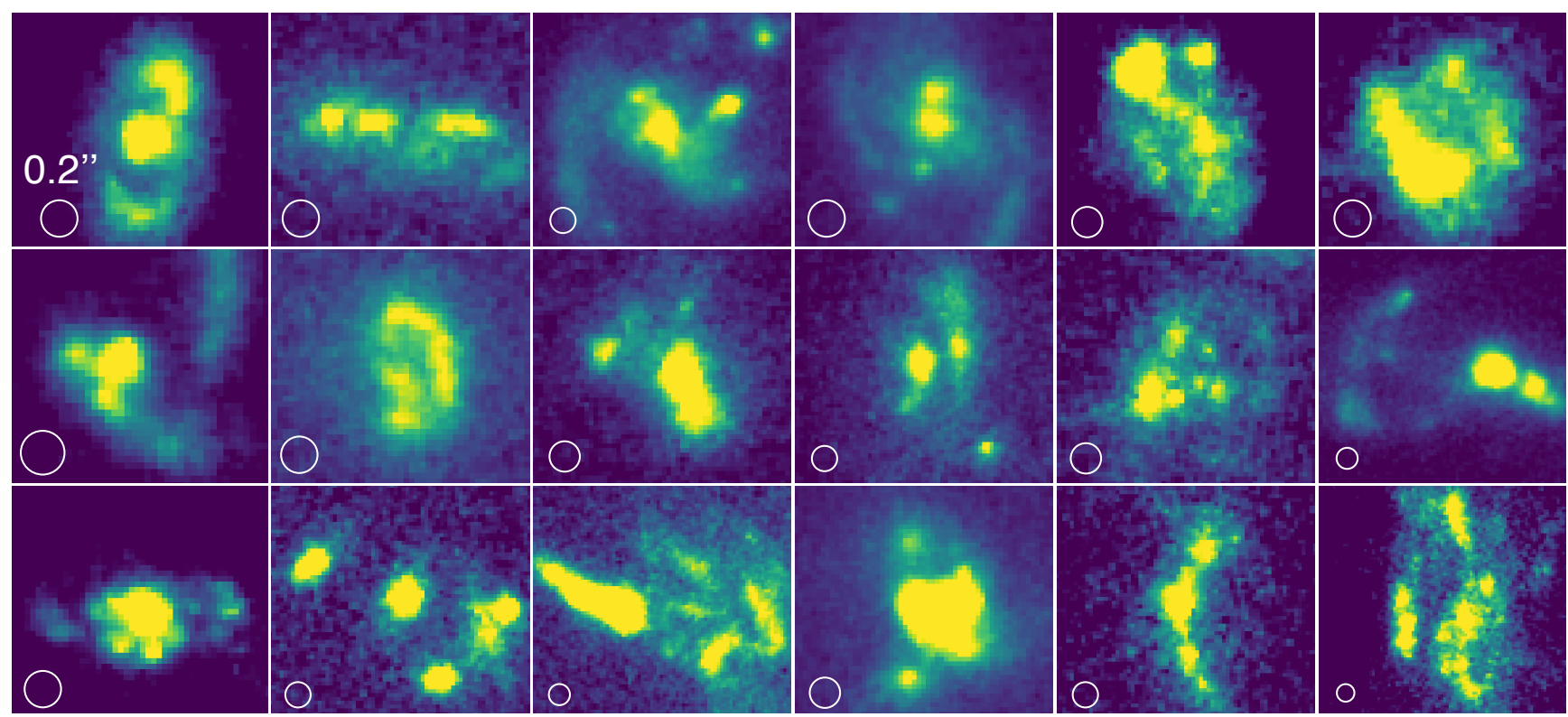

Fig. 1. Representative sample of clumpy starbursts at $0.5<z<0.9$ in COSMOS field, observed by HST-ACS in F814W band. The white circles in the bottom left corner have a diameter of $0.2^{\prime \prime}$.

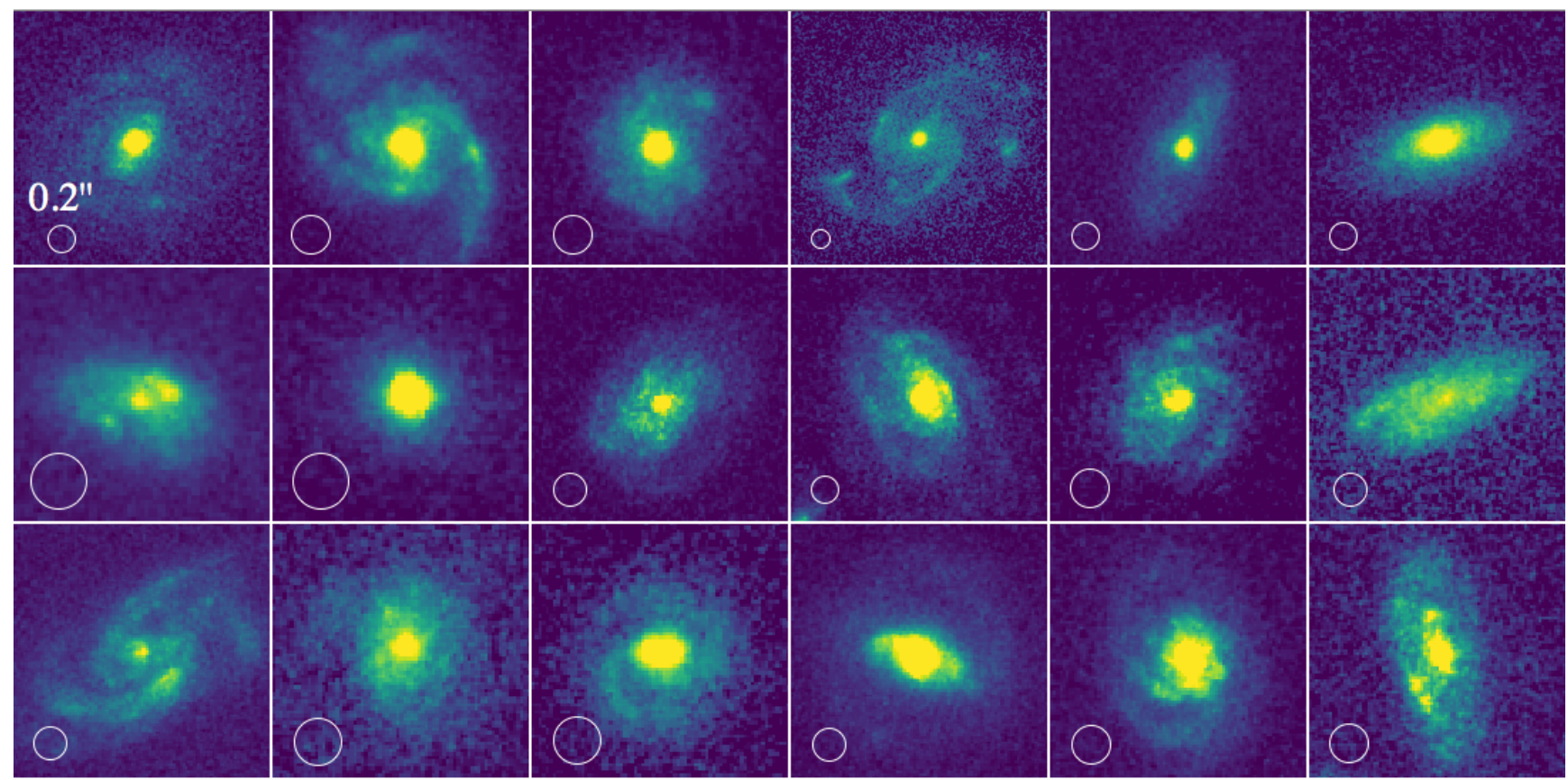

Fig. 2. Representative sample of typical (randomly selected) main sequence disks at $0.5<z<0.9$ in COSMOS field. The image details are the same of Fig. 1.

dynamics of the disks (e.g., rotation direction), and the extinction. For example, the Gini parameter is more sensitive to faceon systems (Lotz et al. 2008). In addition, the surface brightness at redshift $z>0.5$ decreases by more than one order of magnitude compared to the local Universe, making more difficult for interacting signatures to emerge from the noise. All these mechanisms are thus likely responsible for the fraction of lowmergerness SBs identified among our sample.

\subsection{Visual classification}

In order to mitigate previous effects and select a more complete subset of merging systems, we also performed a visual classification. Based on a one-by-one inspection of MS and SB galaxies, we flagged as "visual mergers" all the systems with a disturbed morphology because of the presence of clear tidal tails, shells, bridges, or collisional rings. In addition, we included in this class all the pairs within a projected separation of $<20 \mathrm{kpc}$ and photometric redshift difference $<0.08$. Compared to the previous criterion, this likely identifies mergers on a longer timescale. On the one hand, the pairs select a sample of very early stage mergers that are going to coalesce in $\sim 0.5 \mathrm{Gyr}$ or more. On the other hand, visual mergers also comprise already coalesced colliding systems, if residual merger signatures are sufficiently bright to be detected by eye. These features are typically too faint to contribute significantly to enhance the Gini and 

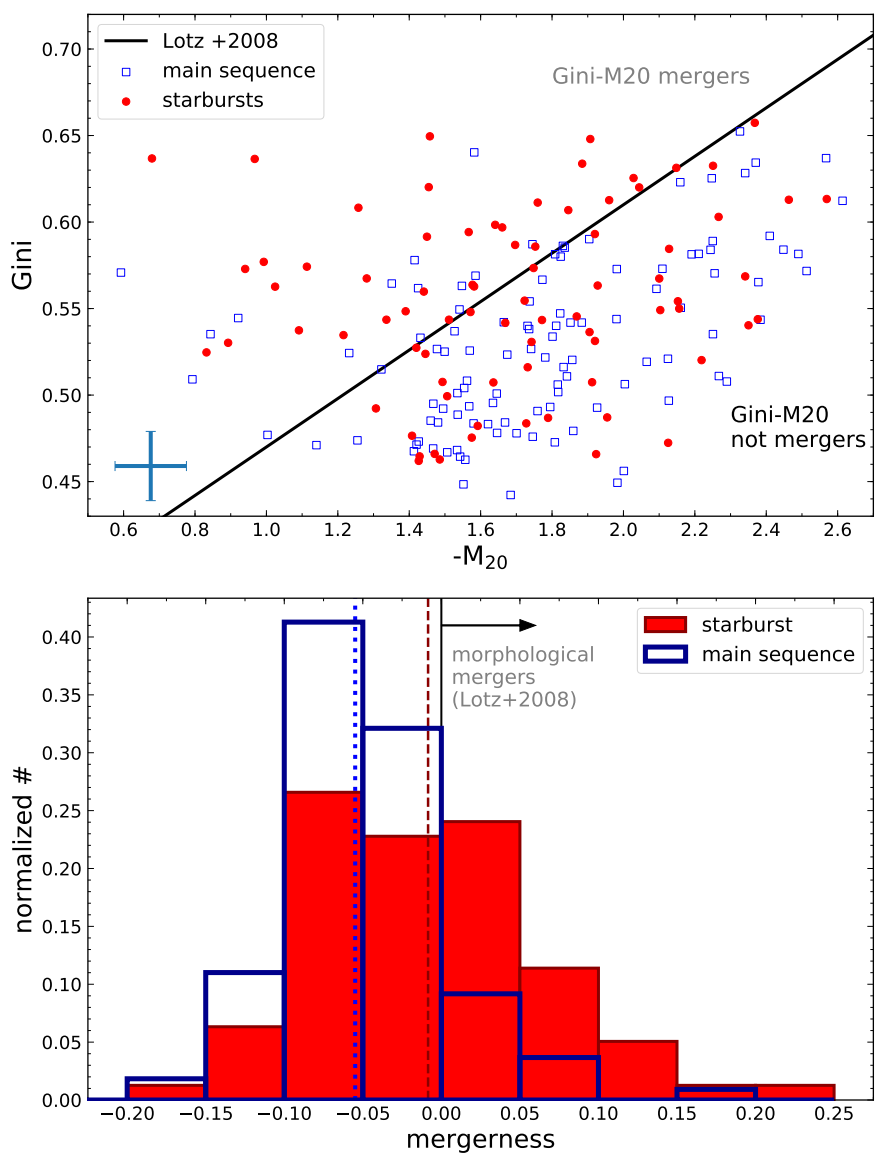

Fig. 3. Top: Gini- $M_{20}$ diagram for the final sample of starburst and main sequence galaxies analyzed in this work (in red filled circles and blue empty squares, respectively). Galaxies above the black continuous line are morphological mergers, according to Lotz et al. (2008). The typical uncertainties of Gini and $M_{20}$ are shown by the representative error bars in the lower left corner. Bottom: histogram distribution (for SBs and MS) of the mergerness parameter, defined as the difference between the measured Gini coefficient and that required to classify the galaxy as a morphological merger. It shows that the majority of galaxies classified as mergers according to this criterion are also starbursts.

$M_{20}$ parameters above the Lotz et al. (2008) limit, so that these mergers are likely missed in the automatic procedure.

This classification yielded a sample of 78 visually selected mergers: 24 of them are in the main sequence $(\sim 20 \%$ of the whole MS population), while the remaining 54 objects $(\sim 68 \%)$ lie in the starburst regime. We also notice that the sample of mergers identified visually is more numerous compared to morphological mergers, which is likely an effect of the longer merger timescale probed by the visual diagnostic, as explained above.

\subsection{Clumpiness measurement}

In order to quantify the contribution of young stellar clumps to the total galaxy emission, we adopted the clumpiness parameter $c$, which measures the fraction of light residing in high spatial frequencies structures. For its estimation, we followed the approach described in Conselice et al. (2003) and Lotz et al. (2004).

Firstly, we smoothed the original ACS F814W images $\left(I_{0}\right)$ using a Gaussian filter with a radius of 5 pixels. This corresponds to an angular scale of $0.15^{\prime \prime}$ and a physical radius of $\simeq 1 \mathrm{kpc}$ at $z \simeq 0.7$ ( 0.9 to $1.16 \mathrm{kpc}$ in our full redshift range), which is the approximate size of the clumps we want to detect (see Sect. 1). Then we subtracted the smoothed image $I_{\text {smoothed }}$ from the original image, imposing 0 for all the pixels with a negative value in the residual image $I_{\text {res }}=I_{0}-I_{\text {smoothed }}$, as done by Conselice et al. (2003).

In a second step, following the procedure adopted in Salmi et al. (2012), we selected all the pixels in $I_{\text {res }}$ with a flux at least $5 \sigma$ above the background of the galaxy, in order to reduce the noise contamination. The background level was estimated with a $\sigma$-clipping statistics procedure applied on all the regions of $I_{0}$ which have not been assigned to any sources in the segmentation map. The threshold limit was chosen empirically, and we found it was the minimum and best value allowing to recover clumpy structures that would have been identified also on a visual inspection. For all the pixels above the $5 \sigma$ threshold, we assigned them a value of 1 ( 0 otherwise), in this way defining a mask for the clumps $\left(M_{\text {clumps }}\right)$. We did not put any constraints on the number of connected pixels to be part of the clumps. However, even when requiring a small amount (e.g., 2-10) of connected pixels, the results are not significantly affected.

In the third step, we removed the galaxy nuclei from the clumpiness calculation, which, by definition, contains only offnuclear clumpy structures. For example, the nuclei of spiral galaxies are usually made of old stellar population bulges that we do not want to consider in the above parameter. For this scope, we derived the segmentation map of the clump mask, deblending the regions containing more than two local luminosity peaks, using the same python codes applied to the original image in the first step. Afterward, we created the nucleus mask $M_{\text {nucleus }}$, setting $M_{\text {nucleus }}=1$ for the clumps identified as nuclei by a visual inspection of the original $i$-band HST images, and 0 otherwise. In most of the cases, the centrally located nuclei correspond to the brightest clumps identified through our routine. However, this is not a necessary condition, since the nuclei (especially the merger cores) can be very obscured and even undetected in optical. Additionally, simulations have shown that the luminosity of newly formed clumps in merger systems can easily overcome that of the nuclei in the two colliding disks. Despite these uncertainties, we conservatively selected and removed at least two nuclei in all the starbursts and in clear MS mergers identified in Sect. 2.2. This represents a limiting case, since we expect that a large fraction of starbursts may actually be fully coalesced systems, even though it is hard to securely isolate them with our data.

Other works systematically mask an inner circle region with a fixed angular aperture for all the galaxies when computing the clumpiness (e.g., Lotz et al. 2004). However, this method may remove clumps that are very close to the nucleus. In addition, it is difficult for any automatic procedure to identify the nucleus, especially in case of disturbed morphologies like in mergers, where a careful visual inspection can be more reliable. For these reasons, we believe that our method is more precise and can be easily kept under control, assuming that the size of our sample is not excessively large. We show in Fig. 4 a representative example (with the galaxy ID 412250) of the full identification procedure of our clumps.

Finally, the clumpiness parameter $c$ was derived in a standard way by dividing the total flux residing in previously detected clumps and the total flux of the galaxy, after masking the nucleus. This calculation can be written explicitly as:

$$
c=\sum_{M_{0} \oplus M_{\text {nucleus }}} \frac{M_{\text {clumps }}(i, j) \times I_{0}(i, j)}{I_{0}(i, j)},
$$




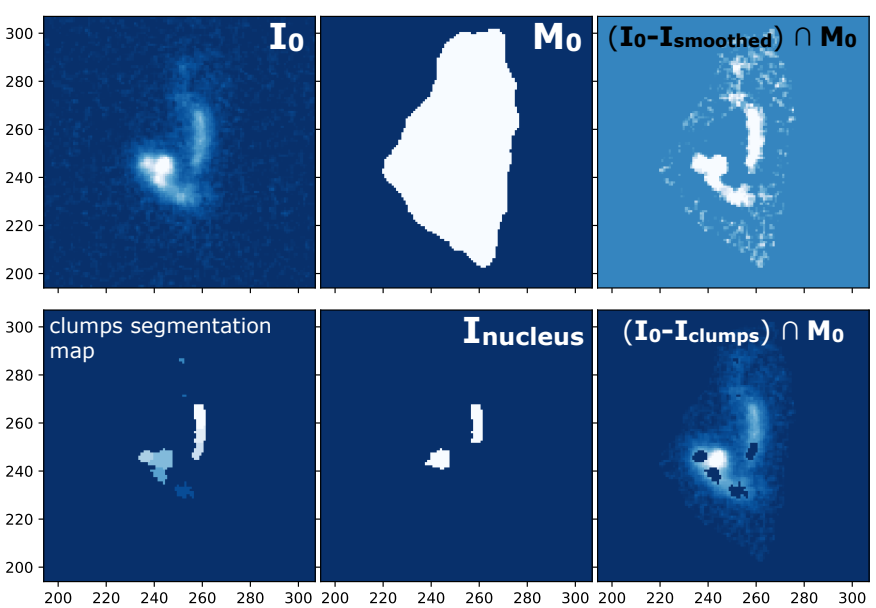

Fig. 4. Flowchart of the clumpiness estimation procedure for one galaxy in our sample (ID 412250): (1) original HST F814W cutout image; (2) segmentation image identifying the galaxy contours; (3) original minus smoothed image, enhancing the visibility of high spatial frequency components; (4) clump detection after applying a $5 \sigma$ threshold (5) nuclei visual identification; (6) residual image after clumps light subtraction, which appear as black regions superimposed to the original galaxy image. This figure highlights the power of our approach. We are able to detect clumps very close to the nucleus that would instead have been removed by masking systematically a circular region around the center. It shows also the important role of the deblending function to separate multiple clumps based on the presence of multiple peaks in a single segmentation region, as explained in the text. This case additionally illustrates our conservative approach: we select two nuclei in the clumps segmentation map even though we are not sure about the second on the right (which in alternative could be part of a tidal tail).

where $I_{0}$ is the original image and $M_{0}, M_{\text {nucleus }}$, and $M_{\text {clumps }}$ are the masks already introduced before. The sum in the formula is done over all the galaxy pixels defined by the segmentation map $\left(M_{0}\right)$, excluding the nucleus. We consider this quantity appropriate for our work, since it compares the flux of the clumps (outside the nucleus by definition) to the total flux in the same off-nuclear regions.

Another viable option would be to compare the emission residing in high spatial frequencies to the total object emission (including the nucleus) as: $c^{\prime}=\sum\left[\left|M_{\text {nucleus }}(i, j)-1\right| \times\right.$ $\left.M_{\text {clumps }}(i, j) \times I_{0}(i, j)\right] / \sum I_{0}(i, j)$, where the sum is over $M_{0}$. For clarity, since this would not change the results of our analysis, we adopt uniquely the definition in Eq. (4) throughout the paper, and include both quantities $c$ and $c^{\prime}$ in Table A.1.

\subsection{Magnitude and elongation cuts: building the final sample}

At $z \sim 0.5$, the brightness of all the objects is approximately one order of magnitude lower (at fixed luminosity) compared to the local Universe, thus it becomes increasingly difficult at higher redshifts to identify the internal structure of galaxies, such as clumps. Moreover, when galaxies become too faint, the visibility and detection of the clumps is automatically affected, so that our method returns systematically lower values of $c$, close to 0 . For this reason, we used the $i$-band magnitudes of Laigle et al. (2016) and applied a threshold as $i_{\mathrm{mag}}<22.5$, beyond which the average clumpiness of galaxies (computed in bins of 0.5 in $i_{\text {mag }}$ ) drops by $>50 \%$ compared to the median value and becomes closer to zero (see Fig. A.1). In addition, for all the galaxies with $i_{\text {mag }}>22.5$, it was harder to distinguish their internal morphology on a visual inspection.
Another important aspect that hampers clumps identification is the inclination of the galaxy. For an edge-on system, the standard detection method can erroneously consider the whole disk as a single elongated (and eventually multiply deblended) clump, producing an artificial enhancement of the clumpiness up to a value close to 1 . In some cases, the edge-on disk is very faint, probably attenuated by the increased dust column density along the line of sight, so that no substructures are detected, and the clumpiness is 0 . However, the majority of these objects would be removed by the first $i_{\text {mag }}$ threshold.

In order to avoid all these cases, we performed a visual inspection and removed all the edge-on galaxies that suffered from these problems. Since the inclination effects can be different for each galaxy and both reduce or enhance their clumpiness, it was not possible to apply the same threshold procedure used for $i_{\text {mag }}$. However, we found that almost all (96\%) of the objects discarded by eye have an elongation $>3.5$, hence we can consider this value as a representative threshold for our selection. For the full original sample, a comparison between the clumpiness and both the $i$-band magnitude and the elongation is shown in Appendix A.

We remark that the $i_{\text {mag }}$ and elongation cuts remove similar fractions of starburst and main sequence systems $(\sim 10 \%$ in each case), and also the same percentages of morphologically classified mergers and not mergers (according to Gini- $M_{20}$ diagram), thus no systematic biases are introduced against either of the two populations. We also notice that almost all of the objects removed by this procedure have very low clumpiness, below 0.05. After applying these cuts, starburst and main sequence galaxies have similar histogram distributions in $i$-band magnitude and elongation in the allowed ranges, and very close medians for the two quantities. In particular, for SBs and MS galaxies, the medians $i_{\text {mag }}$ are 21.25 and 21.35 , respectively, while the median elongations are 1.55 and 1.59 . In any case, we verified that applying lower, more conservative thresholds in $i_{\text {mag }}$ and elongation would not alter the conclusions of this paper.

After cleaning the sample from the contamination of faint or very elongated objects, we derived a final subset of 79 starbursts and 109 main sequence galaxies, which we analyze in the following sections. The final selection of the starbursts and main sequence sample can be visualized in Fig. 5. The stellar mass histogram, visible on the top of the figure, shows that the two selected populations have similar distributions of $M_{*}$, with medians of $\log _{10}\left(M_{*}\right)=10.42$ and 10.39 , respectively, thus our cuts do not introduce systematic biases in $M_{*}$ against one of the two subsets.

\section{Results}

In this section we present the results of our clumpiness measurements, and compare the properties of the starburst and main sequence populations that we have taken as representatives of two star-formation modes: a higher efficiency stellar production induced by merger events in the first case, and a normal starformation activity associated with secularly evolving disks in the latter.

In Fig. 6 we show the histogram distribution of the clumpiness for starburst galaxies (in red) and main sequence galaxies (in blue). The clumpiness parameter spans a range between 0 and 0.20 , meaning that clumps can contribute at maximum to one fifth of the total off-nuclear galaxy emission at this redshift. We can see that the distributions of both subsets are peaked at low clumpiness $(c<0.02)$ and, after this excess, they follow an approximately constant and then declining trend. However, 

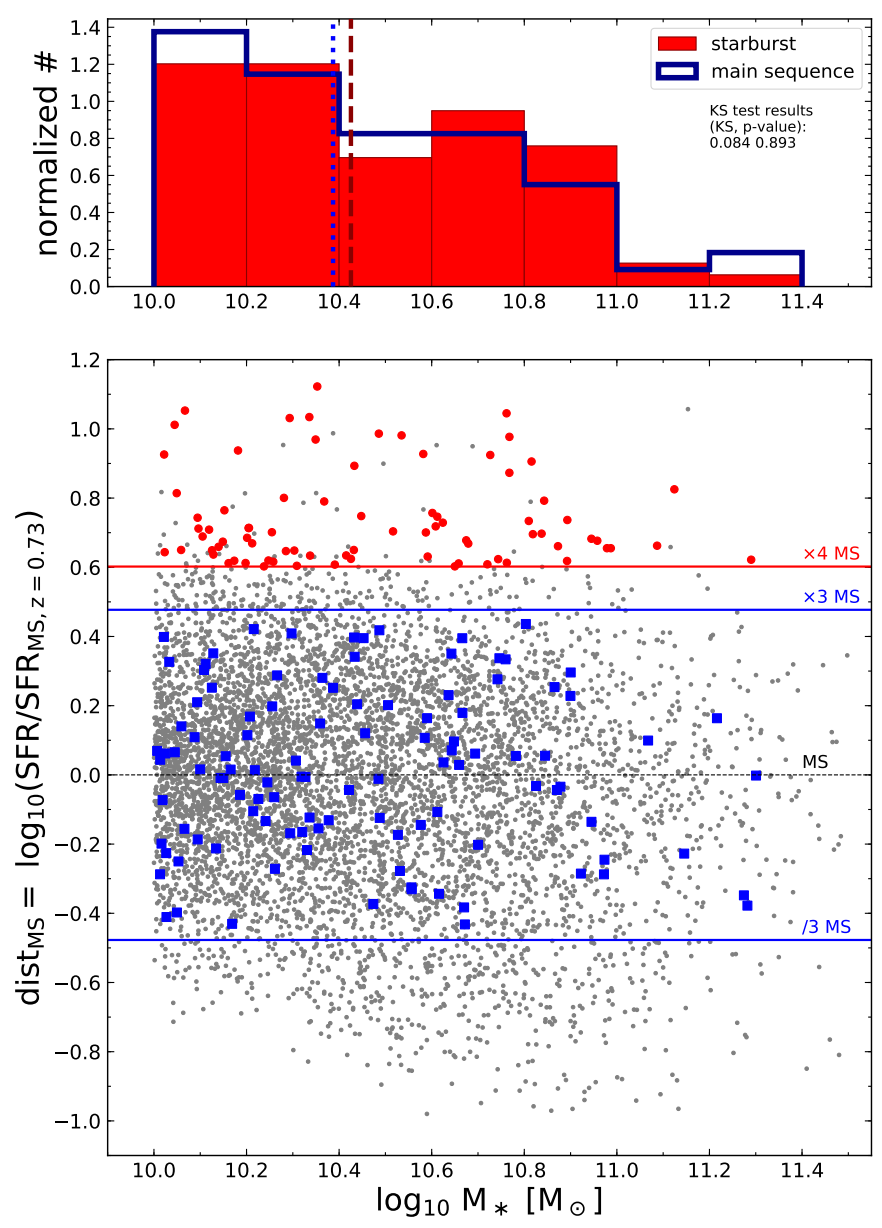

Fig. 5. Bottom: diagram showing the distance from the main sequence as a function of stellar mass for our parent sample of star-forming galaxies in COSMOS $(0.5<z<0.9)$. We highlight with a black horizontal line the 0 level. The blue and red lines indicate the limits taken for our main sequence and starburst selection, respectively. The final selected sample is highlighted with blue squares and red circles, correspondingly. The SFR used in the $y$-axis is normalized to the median redshift of our sample (0.73), following the SFR-redshift evolution of Sargent et al. (2014). Top: histogram distribution (normalized to unit area) of the stellar masses of our selected sample of starbursts (red) and main sequence galaxies (blue), showing that the two subsets have similar distributions. The two vertical lines indicate, according to their corresponding colors, the median values of $M_{*}$ of SB and MS galaxies.

the two histograms differ for many aspects. Main sequence systems are dominant in the first clumpiness bin, while, after a region where the (normalized) number counts are similar $(0.02<$ $c<0.08$ ), starbursts are systematically more numerous above $c \simeq 0.08$. This translates into a median clumpiness for SBs that is more than a factor of two higher compared to the MS population ( 0.05 and 0.022 , respectively).

In order to test whether the two distributions $\left(\chi_{1}\right.$ and $\left.\chi_{2}\right)$ are significantly different, we ran a Kolmogorov-Smirnov (KS) test, which yields both the maximum difference $D_{\chi_{1}, \chi_{2}}$ between the two cumulative distribution functions and the probability ( $p$-value) to obtain the same $D_{\chi_{1}, \chi_{2}}$ under the assumption that the two underlying one-dimensional probability distributions are equal. We found a $D_{\chi_{1}, \chi_{2}}=0.26$ and $p$-value of $0.029 \%$, thus the identity hypothesis can be rejected at $>99.97 \%$ confidence level, suggesting that the two subsets are intrinsically different. We can also characterize the two tails in the high clumpiness regime (excluding the objects falling in the first bin) for SB and MS galaxies separately. The first

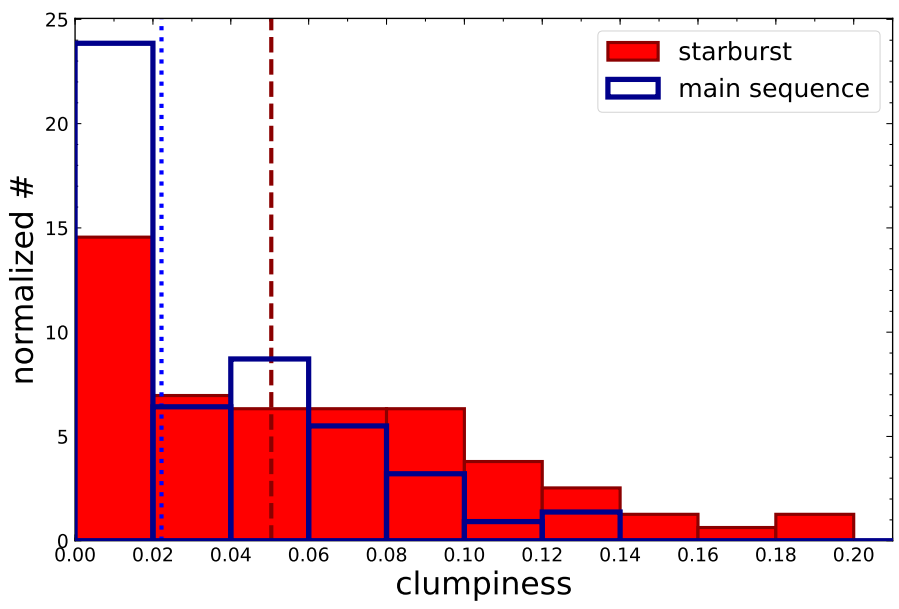

Fig. 6. Histogram distribution of the clumpiness parameter (normalized to unit area) for starburst galaxies (red filled) and main sequence systems (blue edges). This shows that starbursts dominate in the high clumpiness tail. The results of the Kolmogorov-Smirnov test (KS statistics and $p$-value) between the two sample distributions are 0.26 and 0.0029 , respectively. The median clumpiness for SBs and MS $(0.05$ and 0.022 ) are drawn with dashed and dotted lines, respectively, with their corresponding colors.

follows a smooth decreasing trend, reaching a maximum clumpiness of 0.194 , while $c=0.14$ comprises $90 \%$ of the starbursts in the tail. On the other hand, for main sequence systems, the highest clumpiness observed is 0.126 , and, in this case, $90 \%$ of the population in the tail has $c<0.1$.

The detailed properties of the clumpiness distribution among SB and MS systems in our sample can be better visualized in a scatter plot (Fig. 7) by comparing the clumpiness to the distance from the main sequence (dist ${ }_{\mathrm{MS}}$ ). By definition, starbursts occupy the right part of the diagram at dist $_{M S}>0.6$, while main sequence objects span the range between -0.47 and +0.47 . For the whole sample, we also computed the median clumpiness (shown with gray squares) in seven bins of dist ${ }_{M S}$ with bin size of $\simeq 0.2 \mathrm{dex}$, and the error on the median (shown with black symmetric error bars). We also flagged all galaxies with mergerness $>0$ as empty circles, and the remaining not merging systems with black filled circles.

Overall, we can see that there is a large diversity among the main sequence population. Galaxies with dist $_{\mathrm{MS}}<-0.1$ are mostly isolated galaxies, with a low median clumpiness of $\simeq 0.01$ and a maximum value of 0.05 . Above that threshold, a different behavior of the clumpiness can be noticed between Gini- $M_{20}$ mergers and not merger systems. In particular, the clumpiness of the first subset rises on average by a factor of six within the main sequence, and up to a factor of nine at dist $_{\mathrm{MS}}>0.6$. A statistical analysis reveals the existence of a significant correlation between the two quantities, with a Pearson correlation coefficient of 0.39 and $p$-value $=0.006$, although it may not be very strong $(\sim 2.5 \sigma)$ considering the $\mathrm{S} / \mathrm{N}$ of the best-fit angular coefficient. We can also see that the highest clumpiness values $(c>0.14)$ in Figs. 6 and 7 are found only for starburst classified mergers. Interestingly, this upward trend mimics the increase of the morphological merger fraction. Indeed, as showed in the upper histogram in Fig. 7, the relative number of Gini- $M_{20}$ mergers rises along the main sequence, and then goes from $35 \%$ to $\sim 100 \%$ at the rightmost extreme of the starburst regime.

On the other hand, galaxies that are not identified as morphological mergers follow a different trend and do not display 

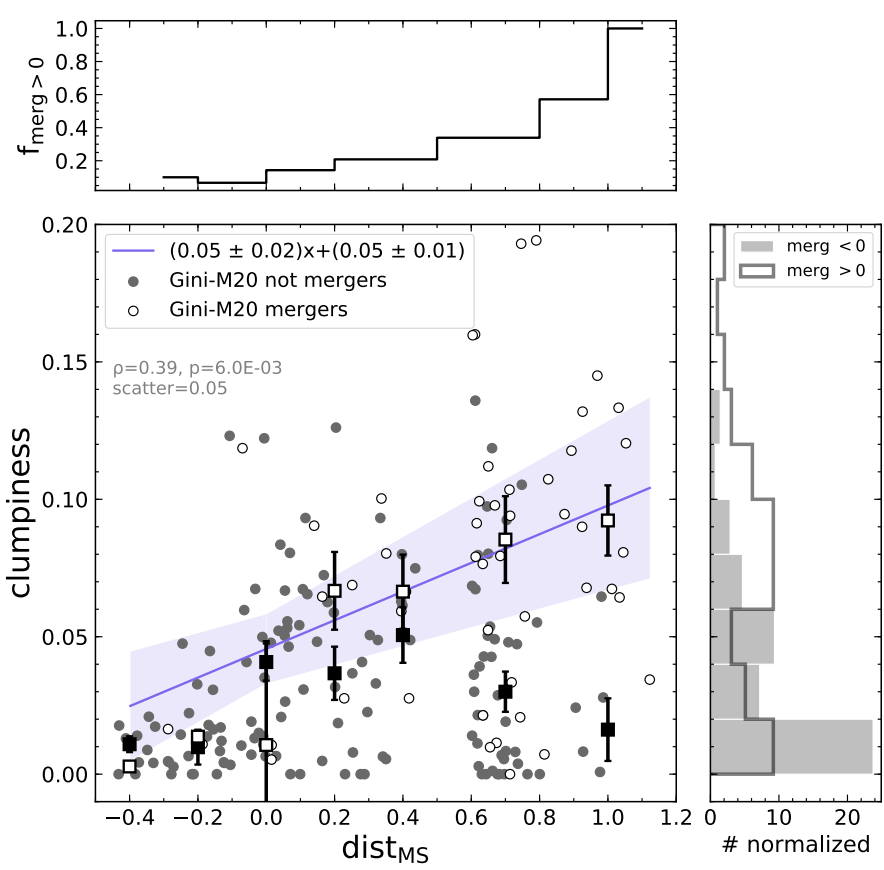

Fig. 7. Scatter plot between the clumpiness and the distance from the main sequence ( $c$ vs. dist ${ }_{\mathrm{MS}}$ ) for our sample. Each galaxy in the plot is color-coded according to its mergerness value. Morphological mergers identified in the Gini- $M_{20}$ diagram (mergerness $>0$ ) are shown with white filled circles, and their fraction $f_{\text {merg }}$ (compared to the whole population) constantly increases toward higher dist ${ }_{\mathrm{MS}}$ (upper histogram). Median clumpiness values and $1 \sigma$ errors are calculated for mergers and not merger systems in seven bins of dist ${ }_{\mathrm{MS}}$ of 0.2 dex approximately. The violet line and shaded area indicate, respectively, the best-fit linear correlation for Gini- $M_{20}$ mergers (whose equation is reported in the legend) and the corresponding $1 \sigma$ error region. The annotation below the legend indicates the Pearson correlation coefficient $\rho$ and $p$-value for the same galaxies, along with the $1 \sigma$ scatter of morphological mergers around the best-fit line. The histogram on the left compares instead the clumpiness distribution for morphologically selected mergers (mergerness $>0$, in white) and not mergers (gray region, derived from the black circles in the scatter plot). In this case, a larger discrepancy is observed between the two distributions compared to Fig. 6, confirmed by the higher significance of the $\mathrm{KS}$ test $\left(\mathrm{KS}=0.432, p\right.$-value $\left.=2 \times 10^{-8}\right)$.

any correlation. In particular, their median clumpiness increases by a factor of $\sim 4$ in the first five bins of dist ${ }_{\mathrm{MS}}$, and stays always between 0.02 and 0.05 in the "upper main sequence" part. Finally, it decreases at dist ${ }_{\mathrm{MS}}>0.6$, even though we have less statistics in the last bin. Similar trends are also found when comparing the clumpiness to the specific SFR.

If we select galaxies according to their mergerness parameter and compare their clumpiness distributions (right histogram in Fig. 7), we find a more clear separation compared to the previous histograms $\left(\mathrm{KS}=0.432, p\right.$-value $\left.=2 \times 10^{-8}\right)$. Interestingly, now, the clumpiness distribution of morphological mergers is approximately constant over the whole range up to $c \simeq 0.12$, since the counts in all the bins are consistent with poissonian statistic fluctuations with $95 \%$ confidence level. If we consider in the same class MS visual mergers and starburst galaxies, and compare them to isolated, not visually interacting main sequence systems, we obtain a similar result of Fig. $7(\mathrm{KS}=0.307, p$-value $\simeq 0.1 \%)$, with the latter prevailing at $c<0.06$ and starbursts dominating at higher clumpiness.

The impact of the morphological merger classification on the clumpiness distributions suggests that we can decompose the entire population in four classes according to their mergerness
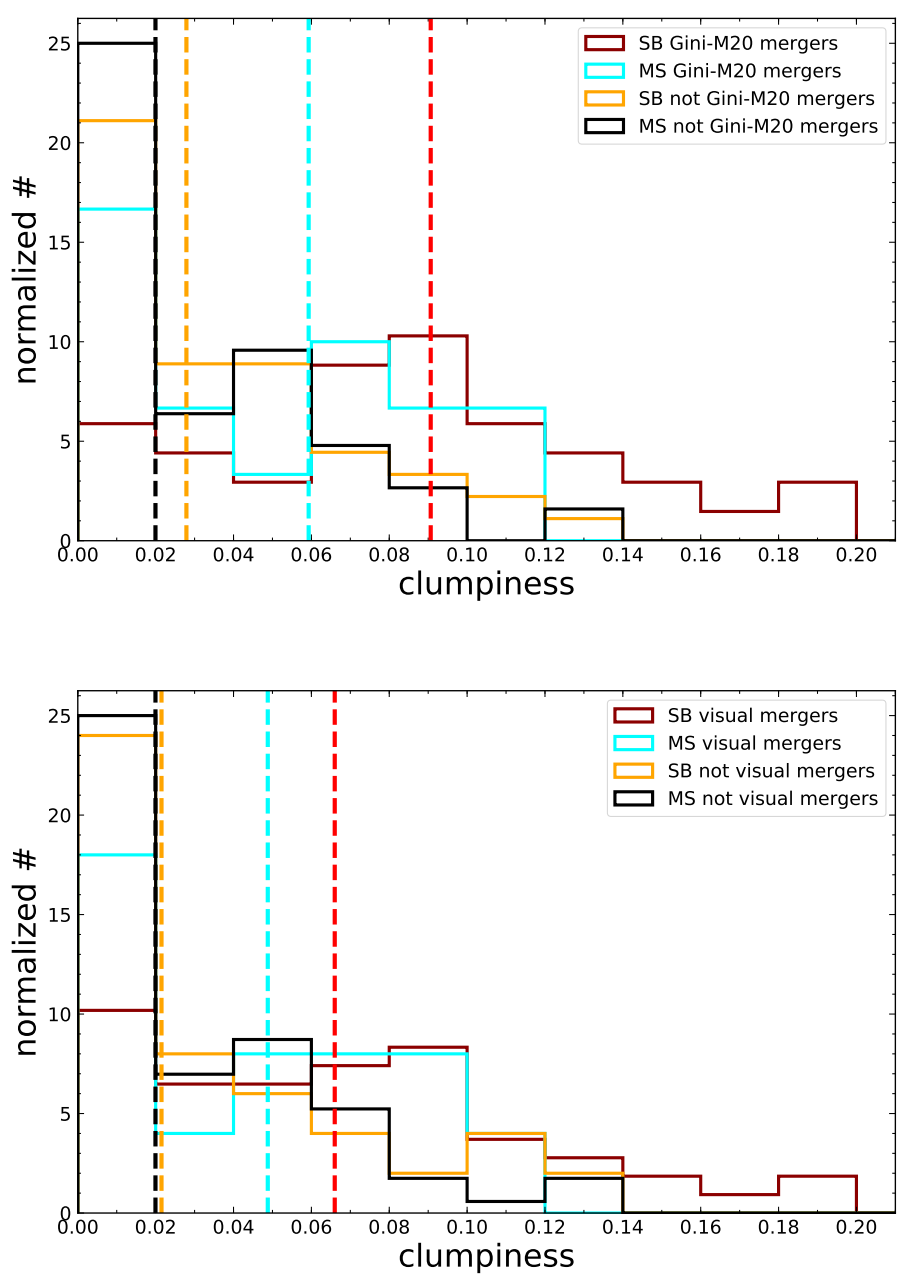

Fig. 8. Top: normalized distribution (continuous line) and medians (vertical dashed lines) of the clumpiness for four types of galaxies: main sequence galaxies with mergerness $<0$ and $>0$ (in black and cyan, respectively), and starbursts (in orange those with mergerness $<0$ and in red the morphological merger subset). We notice that the mergerselected starbursts dominate the high clumpiness tail, while the remaining SBs are basically indistinguishable from the population of main sequence galaxies with mergerness $<0$. A substantial contribution to the high clumpiness population also comes from mergers inside the MS, as shown by the cyan excess at $0.06<c<0.12$. A Kolmogorov-Smirnov (KS) test between merger and not-merger MS systems (cyan and black subsets) yields 0.302 ( $p$-value $=0.094)$. Bottom: same histograms as above, but considering visual mergers instead of the automatic morphological classification.

and dist $_{\mathrm{MS}}$, to look for any trends in the SB and MS subsets. This exercise is made in Fig. 8-top. It shows that, in this case, there is a more striking difference between morphological merger starbursts and not morphological merger systems, regardless of their distance from the MS, the first having a median clumpiness more than a factor of three higher $\left(c_{\text {median }}=0.09\right)$. The clumpiness distribution of MS morphological mergers is also different (at $>90 \%$ confidence level) from the rest of the MS population $(\mathrm{KS}=0.34, p$-value $=0.075)$, with a clumpiness distribution skewed toward a higher values $\left(c_{\text {median }}=0.06\right)$. They also give a large contribution in the higher clumpiness tail (cyan line at $0.06<c<0.12$ ) compared to other not merging systems, suggesting that mergers might increase the clumpiness even with a moderate enhancement of the SFR. A similar result is found when we use the visual classification to identify merger 


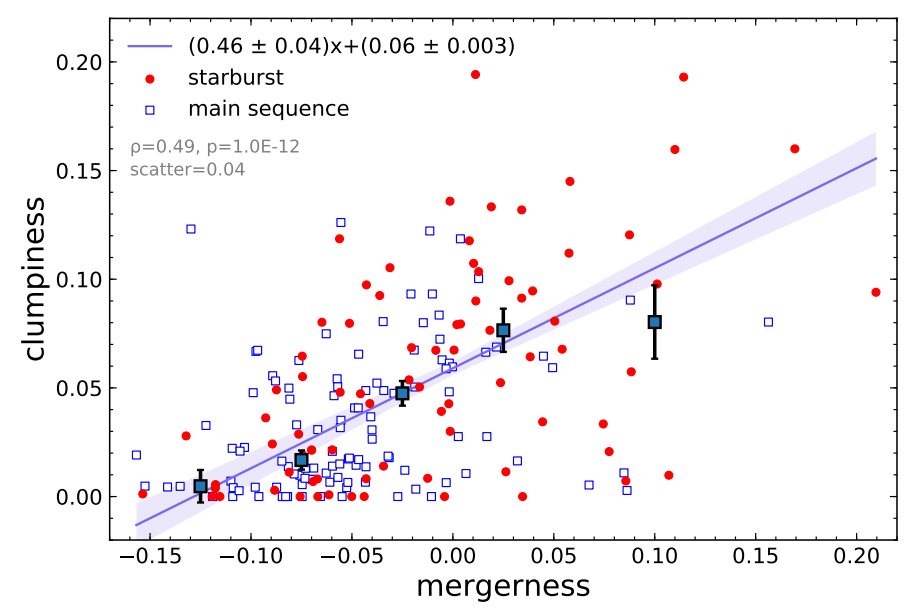

Fig. 9. Comparison between the clumpiness and the mergerness parameters for our sample of SB and MS galaxies (with red filled circles and blue empty squares, respectively). The clumpiness medians with corresponding errors are computed for the whole sample and shown in five different bins of mergerness with square symbols and vertical black bars. The best-fit linear correlation and $1 \sigma$ error are indicated by the violet line and violet shaded area. In the upper-left part of the plot we include the equation of the best-fit line, the Pearson correlation coefficient $\rho$, the $p$-value and the $1 \sigma$ scatter of the relation.

systems in F814W images (Fig. 8-bottom). A KS test performed on visual mergers and not mergers in the main sequence yields a $\mathrm{KS}=0.27$ and $p$-value of $0.1(\simeq 90 \%$ confidence level $)$.

On the other hand, visual merger starbursts are significantly different from isolated undisturbed objects, showing on average higher clumpiness parameters $(\mathrm{KS}=0.38, p$-value $=0.01)$. In this case, the median clumpiness values of merging galaxies (0.066 and 0.049 for SB and MS, respectively) are slightly lower than in the upper panel of Fig. 8, which is somehow expected, given that the two diagnostics probe in general different merger timescales. In particular, as mentioned in Sect. 2.4, visual mergers contain also pairs and already coalesced systems with faint (although unambiguous) residual interacting features, in which the clumps may have still not formed or already disappeared. These objects all contribute to increase the fraction of mergers in the first clumpiness bin $(c<0.05)$.

Given the appreciable clumpiness enhancement in all types of mergers (including MS systems, Fig. 8), we can also check for a possible direct relation between clumpiness and mergerness, comparing these two quantities in Fig. 9. This shows a strong correlation between them (Pearson correlation coefficient $=0.49$, $p$-value $\left.=10^{-12}\right)$, even though with a relatively large dispersion (0.04).

We may wonder whether the two axis presented in Fig. 9 are independent or, in other words, whether the merger classification (i.e., mergerness $>0$ ) is affected by the presence of clumps in the galaxy. However, we have reasons to think that this is not likely the case. First, we do not find a direct relation between the clumpiness and either the Gini parameter or $M_{20}$ separately (Fig. A.2). Even though a slightly increasing trend of the clumpiness is observed on average with higher Gini, many galaxies (both MS and SBs) have a low clumpiness value despite their high $M_{20}$ or Gini coefficients. The presence of clumpy isolated galaxies with relatively high clumpiness but mergerness below 0 indicates itself that clumps cannot be responsible for erroneously selecting the galaxy as a morphological merger. Later in the paper, in Sect. 4.2 (Fig. 14), we corroborate this also through numerical simulations, further showing that the mergerness is independent from the clumpiness. We also remind that our nonparametric morphological procedure is widely tested and validated at all redshifts of our interest (e.g., Lotz et al. 2004, 2008), and we removed faint sources and highly inclined disks for which these tools may not work. Furthermore, we are using multiple merger classifications. In particular, the visual approach classifies galaxies regardless (in principle) of the presence of clumps, and it reinforcing the results found with other methods.

Our findings do not depend significantly on the mergerness threshold used in Sect. 2.3 to identify morphological mergers. To be conservative, if we consider a lower threshold between -0.1 and 0 , this would strengthen the result of Fig. 8, since the median clumpiness of main sequence mergers (cyan vertical line) would move rightward. For example, if we choose a threshold of -0.05 , we would obtain for the above subset a median $c_{\text {med }}=0.048$, and the difference with respect to not merging MS sources would be more significant $(\mathrm{KS}=0.364, p$-value $<0.001)$.

We also remark that these results are not affected by the choice of the bin size and by the sample cuts. Indeed, similar histogram distributions, trends, and significances (Figs. 6-8) are obtained when varying the first parameter by small amounts within a factor of two of the chosen bin size. The same conclusion holds for different thresholds of the $i$-band magnitude for the final sample selection, as in case of more conservative choices (e.g., $i$-mag cut $<22.5$ to keep only the brightest sources).

Furthermore, we have so far analyzed a limiting, very conservative situation, according to which we systematically searched and removed two nuclei in all ongoing merging starbursts. On the contrary, only one nucleus is removed for main sequence galaxies, unless we could clearly see two distinct merging components, in which case we also removed the two nuclei. This approach implies that we are likely underestimating the clumpiness of starburst systems, while simultaneously overestimating that of normal star-forming galaxies, because in many cases one or more of the nuclei might be too obscured to be seen.

Finally, we find no correlation between the stellar mass and the clumpiness (Fig. A.3-top), indicating that the stellar content is not the main driving parameter for the increasing patchy morphology among our sample. Similarly, there is no significant evolution of the clumpiness with redshift, as shown in Fig. A.3-bottom.

\section{Discussion}

Our analysis has shown that merging driven starbursts are significantly more clumpy than normal main sequence galaxies at $z \simeq 0.7$. The question thus arises as to which is the physical reason of this difference. We discuss first the possible role of dust in driving these results, and conclude this is minor. We then use numerical simulations of merger systems at $z \sim 0.7$, showing that mergers can effectively induce clumps formation and increase the measured clumpiness. Later in this section, we support the young nature of the clumps in three galaxies for which multi-wavelength HST images are available. We conclude by discussing a possible merger evolutionary trend of the clumpiness and suggesting a possible extension of our results to interpret clumpy galaxies observed at higher redshifts.

\subsection{Effects of dust attenuation on the observed clumps}

Several studies of the outcoming UV radiation from high redshift clumpy galaxies show that UV clumps may not trace the stellar mass but rather reflect the patchy distribution of dust attenuation (Moody et al. 2014; Cochrane et al. 2019), with FUV emission 
tracing holes in the dust. One may thus ask what is the effect of dust on the clumps that we see in our galaxies.

We remark that our clumps are detected in the optical restframe, at an average wavelength of $4700 \AA$ at $z \sim 0.7$, which is subject to attenuation factors that are significantly lower (by a factor of three or more, assuming for example a Calzetti law with $A_{V}<1.5$ in off-core regions) with respect to the UV regime, on which the above studies are focused. Hence their results cannot be applied straightforward to our case.

Furthermore, our clumps are off-nuclear structures by definition, that is, they are situated several kpc away from the galactic nuclei, which we systematically removed. Hence, they are significantly less attenuated (or nearly unattenuated) compared to the central cores, where most of the IR luminosity is produced. This has been shown in spatially resolved studies of HII regions in massive star-forming galaxies $\left(9.8<\log _{10} M_{*}<11\right)$ at $z \sim 1.4$ (Nelson et al. 2016): outside of the central $1 \mathrm{kpc}$, the attenuation $A_{V}$ decreases at least by a factor of five compared to the center, and is generally low $(\simeq 0.5 \mathrm{mag}$ for optical emission lines). The fact that a subset of our galaxies are highly infrared-luminous is not relevant, as the dusty SFR activity in these systems is mostly concentrated in the central, obscured, usually sub-kpc cores, while the extinction is low in external regions $\left(A_{V}<0.5\right)^{3}$, as shown by spatially resolved studies of local ULIRGs (e.g., Scoville et al. 1997; Alonso-Herrero et al. 2006; García-Marín et al. 2009; Piqueras López et al. 2016).

\subsection{Confirming merger-induced clumps formation with simulations}

In order to check whether mergers are the physical cause of the increased clumpiness, we performed hydrodynamical simulations of collisions, choosing initial conditions that are typical of galaxies in our redshift range. In particular, we set a gas fraction of 30\% (typical of $z \sim 0.7$ ) (Combes et al. 2013; Freundlich et al. 2019). As mentioned in the Introduction, previous simulations of galaxy collisions with such high gas fractions do not reach the low gas temperatures needed to properly reproduce the gas distribution during merger events.

The setup is based on the simulations described in Fensch et al. (2017) using the adaptive mesh refinement code RAMSES (Teyssier 2002). The galaxies have the same characteristics as the ones in Fensch et al. (2017). The refinement strategy is based on the density, and the highest resolution elements are $6 \mathrm{pc}$. Gas in cells that are denser than $10 \mathrm{~cm}^{-3}$ and cooler than $2 \times 10^{4} \mathrm{~K}$ is converted into stars following a Schmidt (1959) law, with an efficiency per free-fall time set to $10 \%$. Three types of stellar feedback are included, as described in Fensch et al. (2017). The energy output from SNII explosions is released by a kinetic kick and a thermal energy injection, each accounting for half of the total energy ouptut (Dubois \& Teyssier 2008). The HII regions are modeled by Strömgren spheres, whose sizes are done considering that the gas surrounding the source has a minimal density above $300 \mathrm{~cm}^{-3}$. Gas inside the sphere is heated to $5 \times 10^{4} \mathrm{~K}$ and receives a radial velocity kick modeling the radiation pressure.

We performed one isolated and two merger simulations. To account for numerical diffusion effect, in the isolated simulation the galaxy moves along the same orbit as one of the galaxies

\footnotetext{
If the real cores were completely obscured in the optical, we could conservatively remove at least two nuclei in starburst objects (and MS mergers) when computing the total flux in clumps, and thus the intrinsic clumpiness would be even higher, which reinforces our conclusions.
}

in the interaction orbits. The spin-orbit coupling plays a significant role in the interaction. For instance, only galaxies with spins aligned with that of the interaction, what is called a "prograde coupling", can create tidal tails (see review by Duc \& Renaud 2013). We ran one prograde-prograde (or, equivalently, directdirect) and one retrograde-retrograde encounter. The two orbits correspond to Orbit \#1 from Fensch et al. (2017). After an intermediate apocenter, they coalesce within $\sim 230$ and $\sim 300 \mathrm{Myr}$, respectively. Stellar density maps are shown in panels A and $\mathrm{C}$ of Fig. 10. On it we see the formation of stellar condensations during the interaction, similar to what is observed in collisions at low-redshift (Di Matteo et al. 2008; Renaud et al. 2014; Matsui et al. 2019). Since we want to check the intrinsic clumpiness of the galaxies, we did not include dust in our simulations.

We then mocked HST observations in the F814W filter by assuming $z=0.7$, the median redshift of our sample. We used the Bruzual \& Charlot (2003) stellar evolution model, with solar metallicity and Chabrier (2003) initial mass function. The stars from the initial conditions were given a random age between $500 \mathrm{Myr}$ and $7 \mathrm{Gyr}$ following a uniform law. The image was convolved to the HST resolution, and the noise corresponding to COSMOS field data acquisition was added to the images. The resulting images are shown in panels B and D of Fig. 10.

In the mock observations, the tidal tails created by the merger are not detected anymore. In contrast, bright clumps, corresponding to the blending of star clusters, are clearly visible at the new resolution in several steps of the evolution. Remarkably, some configurations are very similar to the morphology of the observed galaxies. In particular, the eighth cutout closely resembles the galaxy ID 705860 shown in Sect. 2.2 (the second of the third row). This reinforces the interpretation that clumps (including the nuclei) are probably all part of the same "twobody" merger system, even though their physical association is not obvious by eye. Finally, we measured the clumpiness, Gini and $M_{20}$ parameters from the mock images for both collision geometries and for the isolated case, with the same procedure adopted for the observations. The mergerness was also calculated using Eq. (3).

The results on the morphological properties are shown in Fig. 11. In both merger simulations, the system starts from a low Gini parameter and high momentum of light $M_{20}$, because of the presence of two distant galaxy components. After the first pericenter passage (indicated with a P), Gini increases on average, and the track moves toward the top, as the light becomes more widely distributed along the tidal tales and interacting features. Throughout the middle of the interaction, Gini and $M_{20}$ can oscillate depending on the relative position of the two galaxies and the number of close passages, although the system always remains in the merger region of the diagram, also regardless of the clumpiness value. The two main bodies then approach for the last time and finally coalesce, which implies a rapid fading of the interacting signatures, a net decrease of the $M_{20}$ parameter, and a return into the not merging regime, although in a different region compared to the starting point. We remind that the coalescence (indicated with a C) corresponds to the timestep when only one nucleus is recognized in the segmentation map (instead of two). However, the real physical coalescence of the two unresolved cores may happen at slightly later times, even after the simulation interval analyzed here. For the isolated galaxy, we always stay in the not merger region, below the Lotz et al. (2008) separation line. In all cases, we notice that these simulation runs were performed with a particular (although representative) set of initial conditions, such as inclination and impact parameter, thus in the real case we may populate the Gini- $M_{20}$ diagrams in different ways. 

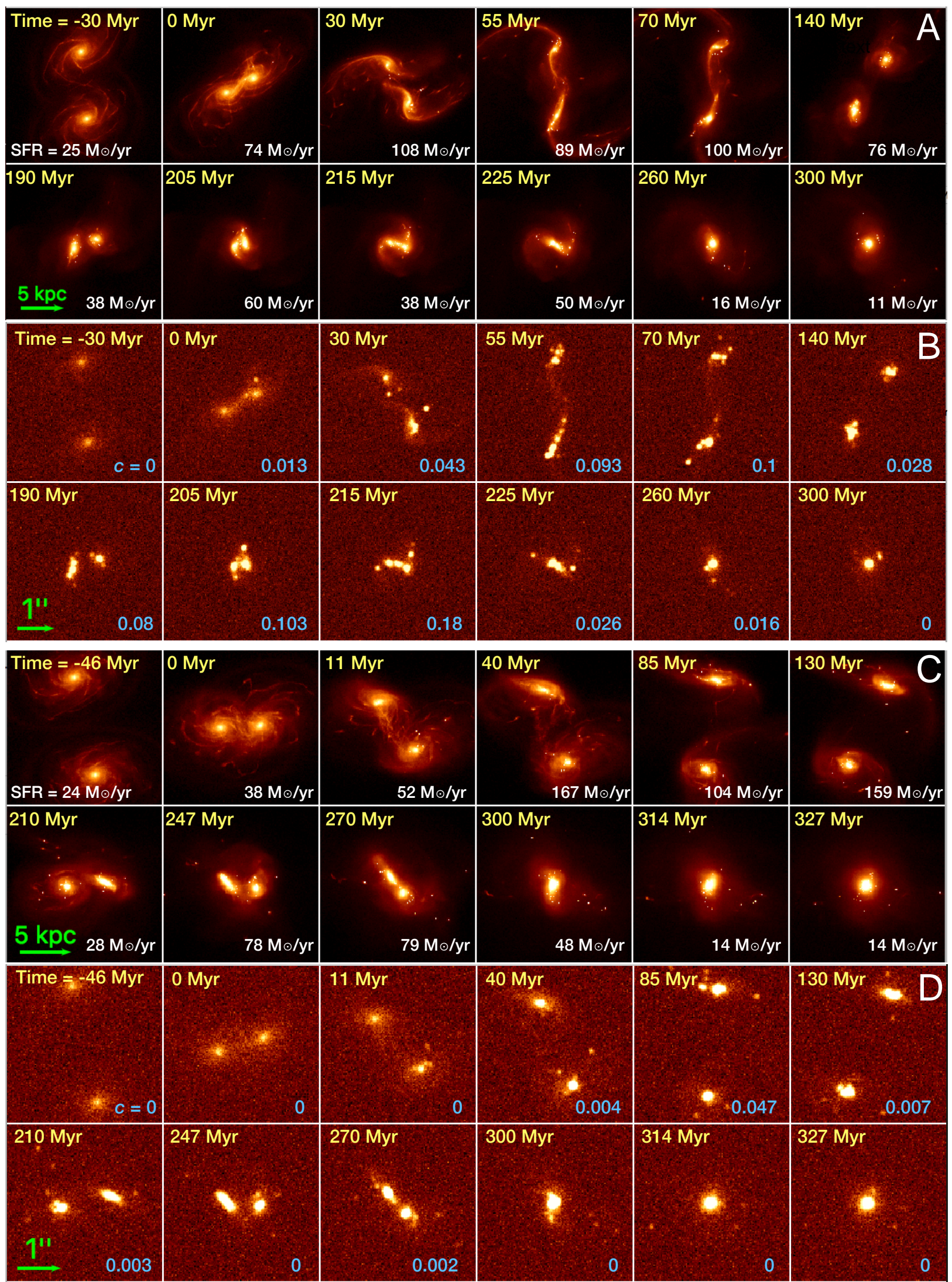

Fig. 10. $A$ : snapshots of the stellar mass density from the simulation of two colliding galaxies with prograde-prograde orbit coupling, at different times. The images have a physical scale of $200 \mathrm{pc} \mathrm{pixel}^{-1}$, comparable to the observations (assuming the median redshift of our galaxies of $\simeq 0.7$ ). $B$ : mock HST F814W observations obtained from the above simulated cutouts after conversion to the $\sim 0.095^{\prime \prime}$ PSF resolution of the images (through a Gaussian filter) and addition of the noise. For each step, the instantaneous SFR and the estimated clumpiness are indicated in the corner. $24 M_{\odot} \mathrm{yr}^{-1}$ is the SFR of two isolated disks, according to the fiducial run. $C$ and $D$ : same as panels A and $\mathrm{B}$, but for a retrograde-retrograde collision. 


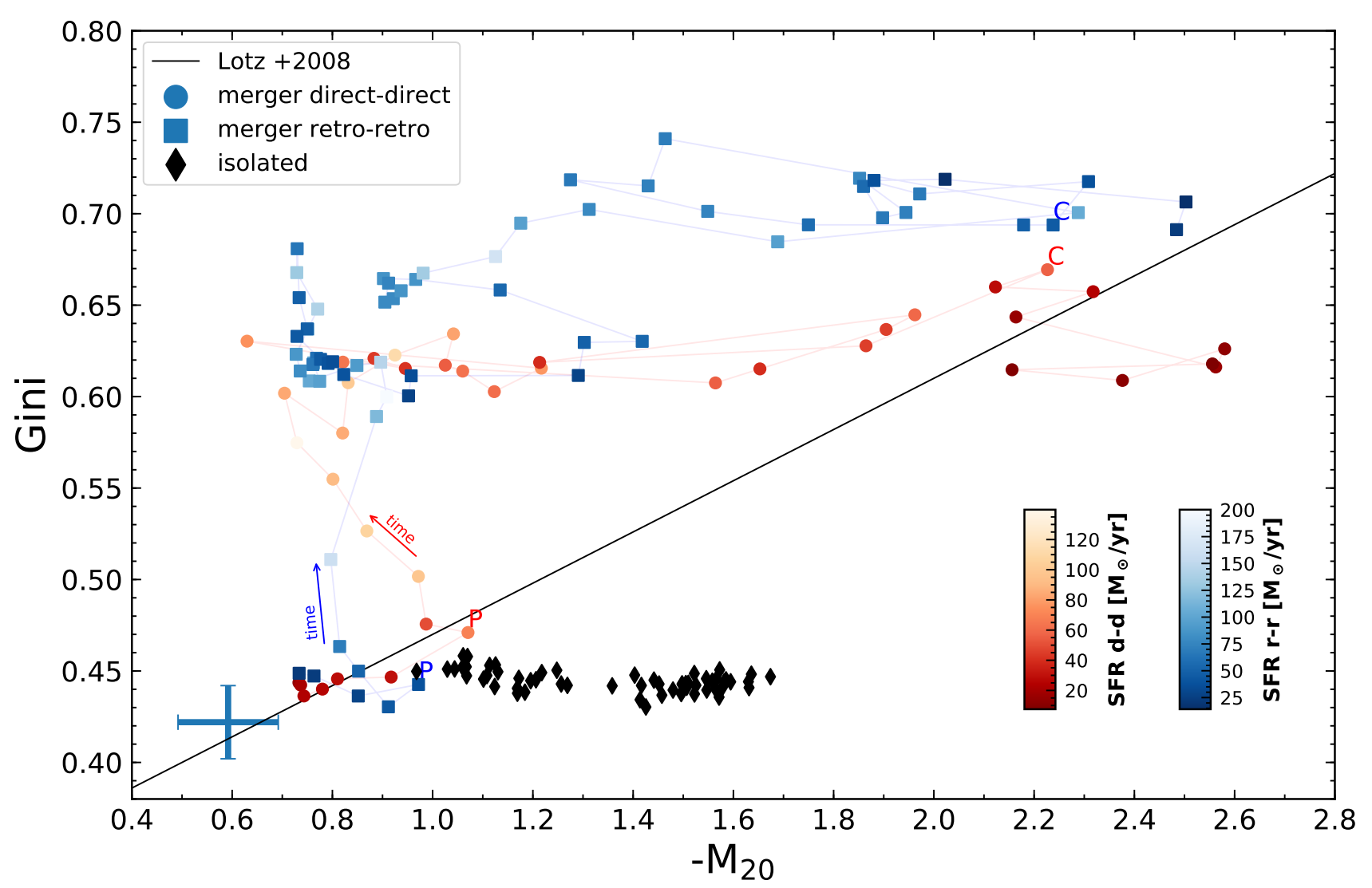

Fig. 11. Time evolution of the Gini and $M_{20}$ parameters for simulated prograde-prograde and retrograde-retrograde collisions (respectively, orange and blue points or connecting lines). The $\mathrm{P}$ and $\mathrm{C}$ symbols indicate, respectively, the first pericenter passage and the coalescence, as defined in the text. We can notice that isolated galaxies spend all their lifetime in the not-merger region (according to the separation line by Lotz et al. 2008), while ongoing merging systems are mostly located above the morphological merger criterion. For each simulation subset, the points are color-coded according to the specific SFR, while black arrows indicate the positive direction of time.

The time evolution of the SFR and the clumpiness for the three simulations is shown instead in Fig. 12. In the upper panel of this figure, we notice a rapid increase of the SFR after the first pericenter passage and at the coalescence, similarly to previous simulations of galaxy collisions. We can also see that the SFR increases by a factor of four to five, which is an intermediate value between the high enhancement $(10-100)$ that can occur with $10 \%$ gas fraction, and the low enhancement obtained for the same orbits and a 60\% gas fraction (below 4) (Fensch et al. 2017). This effect will be discussed in more details in a companion paper (Fensch et al., in prep.). We additionally remark that a time averaging of the SFR (and of the clumpiness) in the past $50 \mathrm{Myr}$ could be applied if we want to match the average SFR timescale of our observations, even though the diagrams would not change qualitatively.

The evolution of the clumpiness is displayed in the bottom panel of Fig. 12. While we see that for both collision orbits the clumpiness increases compared to the fiducial run, the relative values are quite different. The prograde-prograde orbit reaches high values of the clumpiness $50 \mathrm{Myr}$ after the first pericenter and right before the coalescence, and peaks at 0.17 , which is in the range of clumpiness observed in our work. On the contrary, the clumpiness does not increase much during the retrograderetrograde orbit, reaching only 0.05 , at $75 \mathrm{Myr}$ after the first pericenter.

On Fig. 10, we can see that a high clumpiness in the prograde-prograde case is obtained by the blending of star clusters, which tends to happen at the base of the tidal features.
This blending results in the accumulation of star clusters in this region, as we can visualize in the fourth cutout. The formation of the tidal tail resulting from the prograde spin-orbit coupling, the accumulation of star clusters and the enhanced clumpiness do not happen in the retrograde-retrograde collision.

Overall, our numerical simulations are able to explain qualitatively several observational findings. First, the merger simulations can explain the increase of the clumpiness (i.e., the fraction of light coming from clumpy structures) up to values that are similar to those observed. Simulations are also able to reproduce qualitatively the increasing trend of this parameter with specific SFR and mergerness, which we have seen observationally in Figs. 7 and 9, respectively. In addition, the same simulations show that the beginning of the clumpiness enhancement occurs at around the same time of the first rise in SFR, not before. These findings suggest that clump formation and starburst activity in mergers are driven by the same underlying mechanisms, that are, enhanced gravitational instability and collapse due to the merger perturbations.

Secondly, during a merger event, a significant increase of the clumpiness can be obtained even with a modest SFR enhancement, below the threshold of starburst classification (Fig. 13top). This can explain the fraction of morphological mergers in the main sequence with a relatively large clumpiness parameter.

Furthermore, we found that the geometry of the interaction plays an important role. For example, in the case of a retrograderetrograde merger, only a very small enhancement of the clumpiness is found during the starburst, or no increase at all. This 

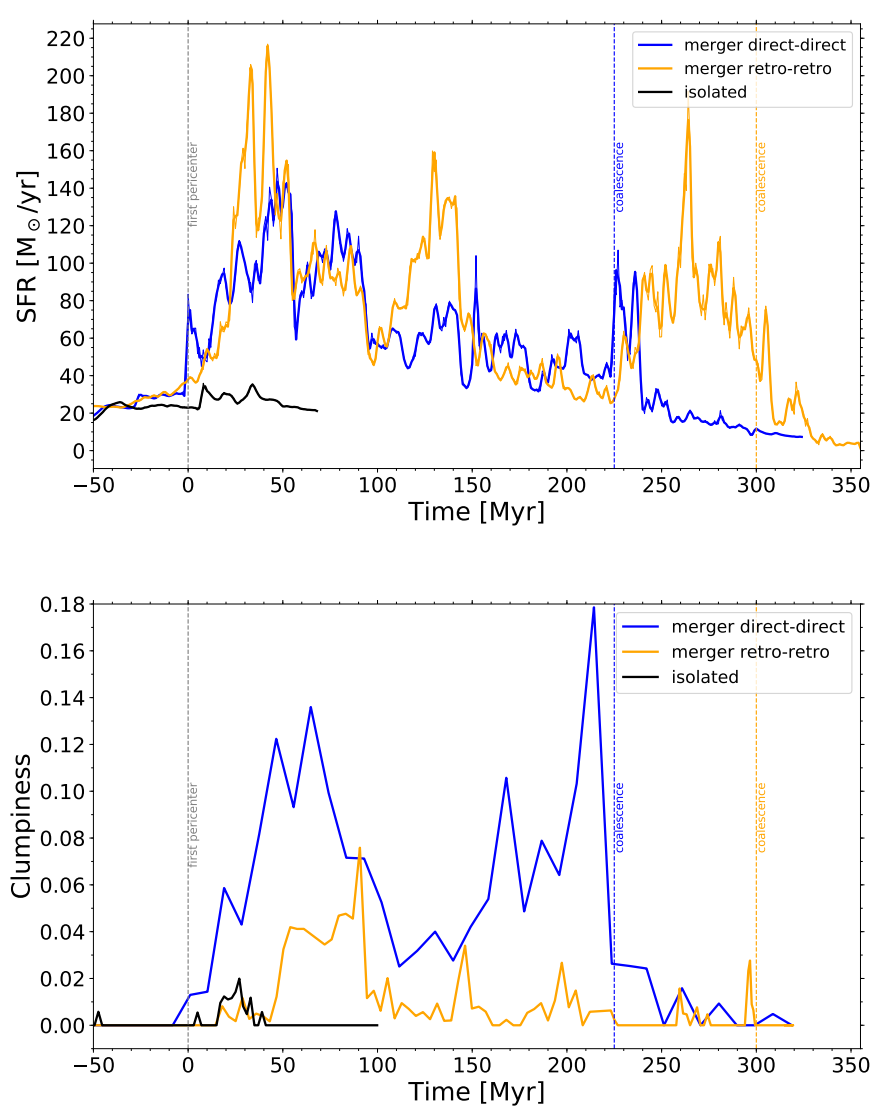

Fig. 12. Top and bottom: SFR and clumpiness (respectively) are shown as a function of time for the direct-direct (i.e., prograde-prograde) merger (in blue), retrograde-retrograde merger (in orange) and for the fiducial run of an isolated galaxy multiplied by two (in black). In the latter, only the evolution during $200 \mathrm{Myr}$ is shown as a representative case, while in the other two runs the time range considered $(\sim 450-\sim 820 \mathrm{Myr}$ from the onset of the simulation) encompasses the effective merging period, from the first pericenter passage to the final coalescence (vertical dashed lines colored accordingly).

implies that a high clumpiness parameter is not obtained neither in all mergers, nor in all starburst systems. We remark that having very low $c(<0.04)$ at elevated SFRs $(\times 4$ above the MS) is not inconsistent with our data (cf. Fig. 6). It is thus clear that the variety of spin-orbit couplings can explain part of the scatter of the clumpiness observed in our sample. A forthcoming paper will study the physical properties and evolution of simulated clumps with more detail, with a more diverse set of galactic disks (Fensch et al., in prep.).

Finally, we may wonder how simulation results would change if we include the dust attenuation. Several works have already done this, showing that the extinction has effects, in particular on the SFR estimates, but not on the existence of clumps themselves (Jonsson et al. 2010; Hayward et al. 2011). Clumps identified in stellar mass maps are still detected in the optical in simulation images of submillimeter-bright galaxies (Cochrane et al. 2019). Furthermore, at higher redshifts $(z \sim 1)$, the extinction is not particularly high even in big UV clumps (regardless of being merger-induced or not), so the patchy radiation that we detect does not represent just holes in the dust distribution, but real clumps with high gas and SFR densities (Elmegreen \& Elmegreen 2005; Wuyts et al. 2012). For these reasons, the dust attenuation would not change the results of our simulations.
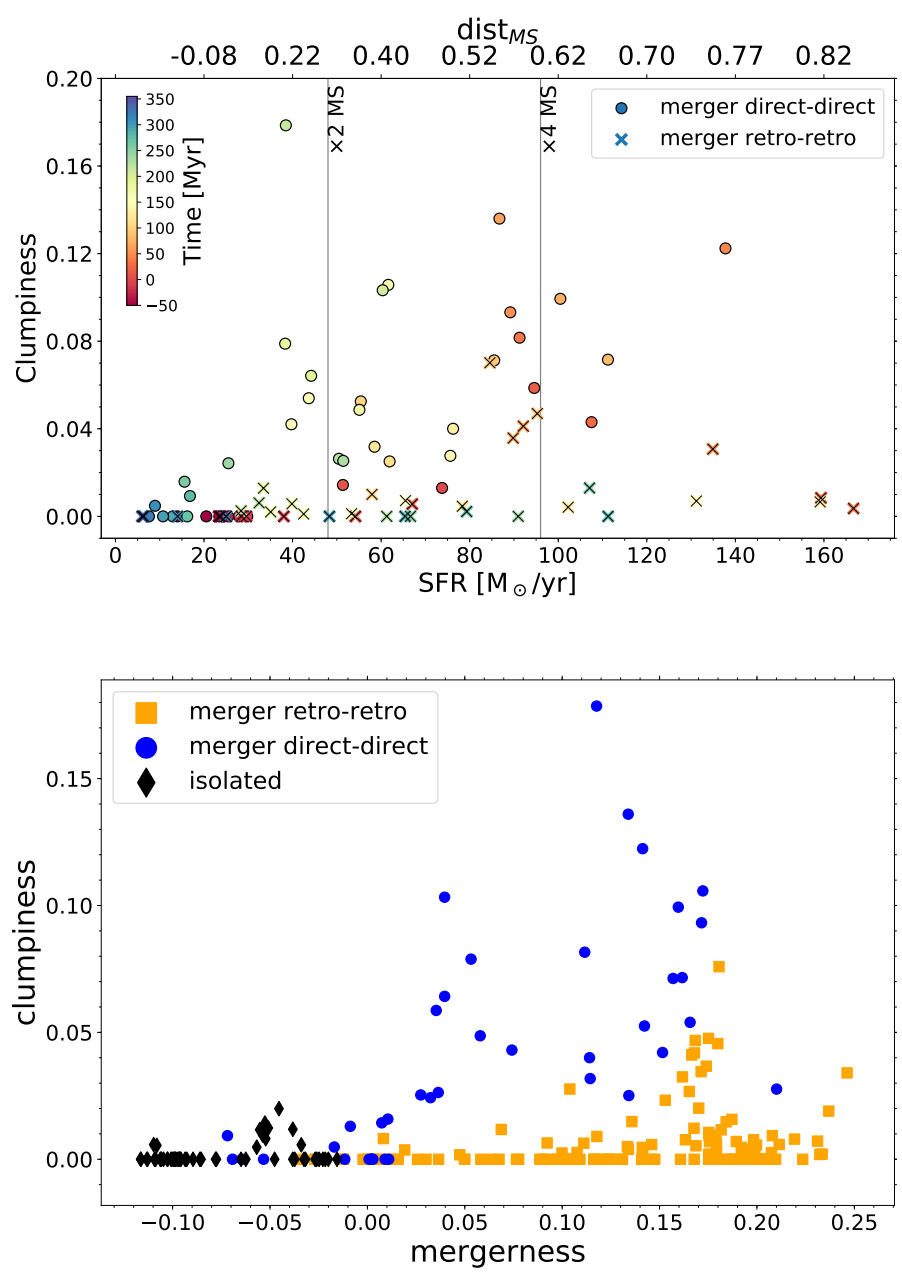

Fig. 13. Top: comparison between the clumpiness and the SFR at different timesteps (colorbar on the left), for direct-direct and retrograderetrograde mergers (with circles and crosses, respectively). This diagram should compared to the corresponding observational result in Fig. 7. Bottom: clumpiness plotted as a function of the mergerness parameter, which is calculated from mock images at finite time intervals. This panel can be compared with the observational finding in Fig. 9. In both cases, we can notice a correlation between the quantities in the $x$ and $y$ axis, which supports the observational results.

\subsection{Clumpiness evolution during the merger}

As shown in Sect. 3, we divided the sample in SBs and MS galaxies, finding that the two subsets have different clumpiness distributions: the former prevail in the high clumpiness end, while the latter dominate at very low $c(<0.02)$. However, this classification is too simplistic. The intrinsic complexity of the MS and SB populations can be resolved in part by considering their apparent morphology. We have indeed seen in Fig. 8 that morphological merger starbursts, selected by their large Gini and $M_{20}$ coefficients, are the major responsible for the high-end tail of the clumpiness distribution, with a median $c=0.09$ and a maximum fraction of light from the clumps of $20 \%$. On the other hand, the subset of starbursts that are not selected as mergers from their morphology is essentially indistinguishable from that of isolated (i.e., not merger) main sequence systems (Fig. 8).

As highlighted in previous works, the morphological classification criterion defined by Lotz et al. (2004) is able to identify mergers over a relatively short temporal window compared to the whole merger duration. The observability timescale of a merger 
in the Gini- $M_{20}$ diagram is approximately $0.2-0.3 \mathrm{Gyr}$, according to Nevin et al. (2019), corresponding to the period when the interaction signatures are more evident in the form of bright tidal tails or very disturbed, elongated, or asymmetric global structures. In contrast, at the coalescence, residual merging features rapidly fade below the surface brightness detection threshold, hampering its true nature recognition visually, especially at our redshifts. This increasing difficulty of identifying mergers at the coalescence is also seen in simulations, which suggests that different time evolutionary merger phases can in part explain the large spread of mergerness and clumpiness in the SB distribution.

In order to test this interpretation, we analyze a subset of 21 starbursts with near-infrared rest-frame spectra (and with available HST images in our redshift range) that have been presented in Calabrò et al. (2018). These galaxies are representatives of off-MS systems at $0.5<z<0.9$ above $M_{*}$ of $10^{10} M_{\odot}$. In Calabrò et al. (2019) we show that this sample comprises a sequence of different evolutionary merger stages, traced by the equivalent width (EW) of Balmer or Paschen lines, and by the total attenuation $\left(A_{V, \text { tot }}\right)$ toward the center of the starburst core in a mixed dust and stars configuration (Calabrò et al. 2018).

In Fig. 14 we compare the clumpiness of this SB subset to $A_{V \text {,tot }}$ and to the EW of $\mathrm{H} \alpha$ and $\mathrm{Pa} \beta$ lines, measured in Calabrò et al. (2019). In the first upper panel, we find no significant correlation between $c$ and $A_{V \text {,tot }}$, as the Spearman correlation coefficient $R=-0.34(p$-value $=0.13)$ and the angular coefficient of the best-fit line is consistent with 0 . However, if we assume no correlation, the four starbursts with the highest obscurations $\left(A_{V, \text { tot }}\right.$ above $\left.15 \mathrm{mag}\right)$ all have a low clumpiness below 0.04 . This confirms that an important fraction of galaxies $(50 \%$ in our Magellan dataset) contributing to the first two low clumpiness bins in Fig. 6 may be actually late stage mergers observed after the coalescence. On the other hand, early and intermediate merger systems with $A_{V \text {,tot }}$ between 0 and 15 can show the full range of clumpiness values. These are also the only phases where we observe a substantial clumpiness enhancement above $10 \%$ and above the average population level.

In analogy to the former result, when comparing the clumpiness to the equivalent width of $\mathrm{Pa} \beta$ and $\mathrm{H} \alpha$, we also measure an angular coefficient slightly below $2 \sigma$. However, in the latter case, the Spearman coefficient $\mathrm{R}$ is equal to $0.53(p$-value $=0.014)$, indicating that the correlation is significant according to this statistical test. The existence of this correlation (even though mild), and the position of the Magellan SBs in the first panel of Fig. 14, can shed light on the possible triggering mechanisms and fate of the observed clumps. Following the results of previous simulations, clumps can form with the increasing compressive turbulence modes and subsequent fragmentation induced by the merger during early-intermediate stages (Renaud et al. 2014). The absence of late stage mergers with high clumpiness suggests that, after the main triggering event, clumps could be rapidly destroyed by strong stellar radiation or AGN feedback, or they could migrate toward the central galactic bulge.

Intriguingly, we notice that four out of five X-ray detected AGNs in this small subset have a very low clumpiness below 0.02. As suggested in Calabrò et al. (2019), these systems may be in an advanced phase of the AGN activity and central black hole growth, possibly approaching the blow-out phase, thus an impact of this feedback activity on the lifetime of clumps cannot be excluded. If we do not consider these objects, we would obtain a higher statistical significance of the $A_{V, \text { tot }}-c$ correlation.

Another possibility is that clumps become too faint in $i$-band compared to the host galaxy, following the aging of the stellar

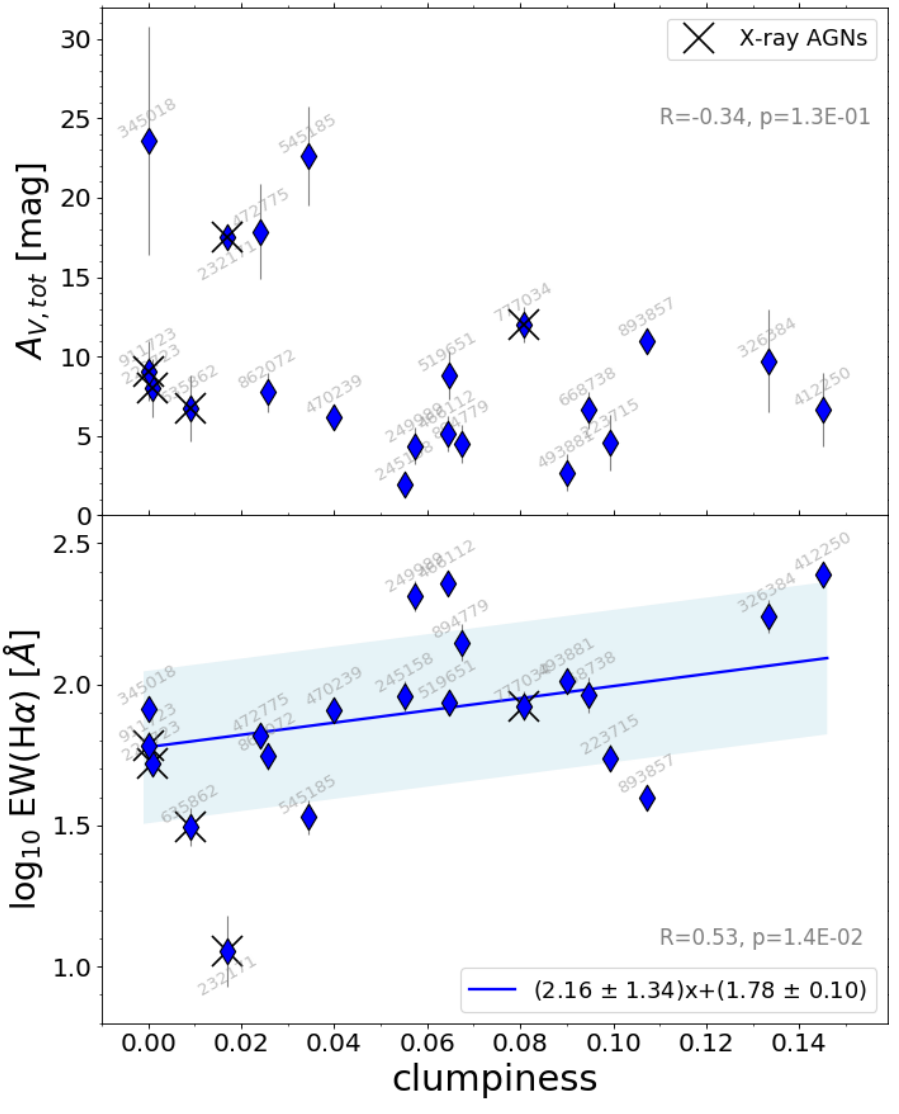

Fig. 14. Comparison between the clumpiness and the mixed-model attenuation $A_{V \text {,tot }}$ toward the center (upper plot), and the equivalent width (EW) of $\mathrm{H} \alpha$ (lower panel) for 21 starbursts in the redshift range 0.5-0.9 analyzed in Calabrò et al. (2019). The corresponding IDs are added to each galaxy, while black crosses indicate X-ray detected sources. The Spearman correlation coefficient $\mathrm{R}$ and the corresponding $p$-value are highlighted in each panel. We derived no correlation for the first diagram and a mild correlation $>3 \sigma$ for the second, thus only for the latter case is shown the best linear fit (blue line, with equation included the legend) and $1 \sigma$ dispersion (blue shaded region). However, even in the first plot, the highest clumpiness values are found only for the less attenuated starbursts, while low clumpiness objects are systematically more obscured $\left(A_{V, \text { tot }}>15\right)$. Given that $A_{V, \text { tot }}$ and $\mathrm{EW}(\mathrm{H} \alpha)$ have been used as merger stage indicators in Calabrò et al. (2019), our findings provide an indication for a possible clumpiness evolution, decreasing from early-intermediate phases to late stage mergers. This is corroborated by the subset of X-ray AGNs (likely close to the blow-out phase) showing preferentially a lower clumpiness.

populations or the higher dust obscurations expected in advanced phases, and they are not detected anymore in this band. However, we remind that testing the impact of feedback processes and studying the final fate of the clumps is beyond the scope of this work, and it will be investigated in future papers.

Even though the time evolutionary sequence is a tantalizing interpretation, the absence of very strong correlations may indicate that other effects also play a role on the formation of clumps, such as the impact geometry, the dynamics and mass ratio of the two components, and the viewing angle toward the system, all of which would be in any case very difficult to quantify from current observations. In particular, the strong dependences on the orbital configuration (Sect. 4.2) suggest that we may have large object by object variations in the clumpiness while having still a strong SFR enhancement (Fig. 13-top). 

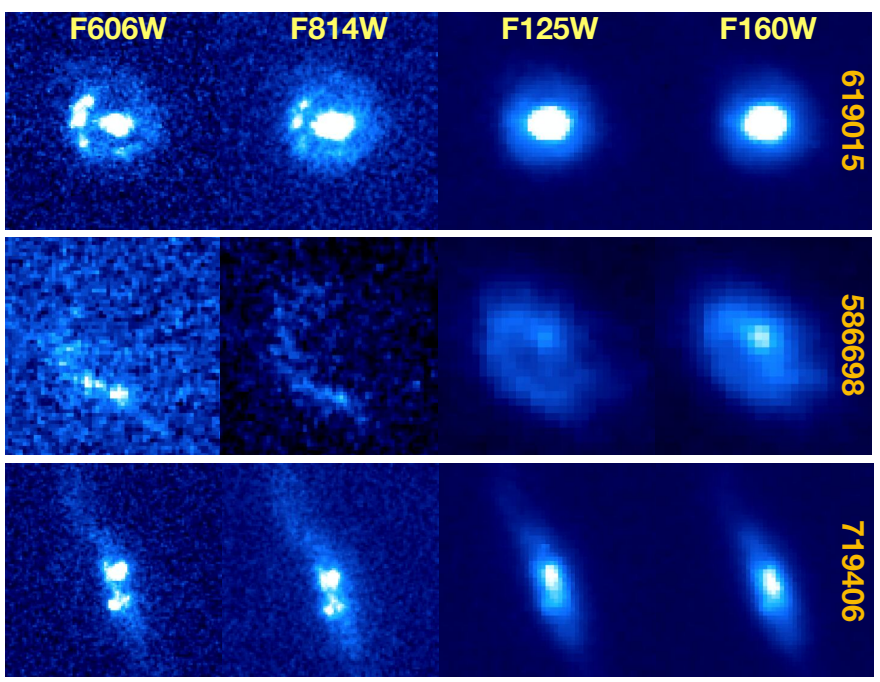

Fig. 15. HST cutout images in the F606W, F814W, F125W and F160W broad band filters for three starbursts in the COSMOS-CANDELS field. As explained in the text, the second galaxy has a photometric redshift of 0.92 , while the third has a SFR a factor of three above the main sequence at the same redshift $z=0.74$, but clearly shows a merger morphology (in spite of its edge-on profile). The multi-wavelength comparison shows that clumps become brighter at shorter wavelengths, thus they likely represent merger-induced young stellar associations rather than old preexisting structures. The IDs shown in the right part of the figure come from Laigle et al. (2016).

\subsection{Multi-wavelength morphology from CANDELS}

We have identified clumps in single broad-band (F814W) images, which are available for the majority of galaxies in the $2 \mathrm{deg}^{2}$ COSMOS field. A possible limitation of this approach relies on the fact that we can probe the emission of clumpy structures only in a limited wavelength range, which, considering the filter transmission curve and our redshift interval, covers approximately the 3700-6400 A rest-frame range. The morphology of clumpy features can potentially change if we go to the UV restframe or at longer wavelengths.

We can check the multi-wavelength morphology of three starburst galaxies in our sample that are included in the COSMOS-CANDELS field, observed at high-resolution by HST (Koekemoer et al. 2011) in four broad-band filters (F160W, $\mathrm{F} 814 \mathrm{~W}, \mathrm{~F} 125 \mathrm{~W}$ and F160W) at resolutions of $0.08^{\prime \prime}, 0.09^{\prime \prime}$, $0.12^{\prime \prime}$, and $0.18^{\prime \prime}$, respectively (Fig. 15). In order to have a larger statistics, we consider in this analysis one galaxy in the subset analyzed so far (ID 619015), and other two galaxies (ID 586698 and 719406) that belong to the parent sample but were excluded from the final selection adopted in this paper. While the first has a slighly higher redshift (0.92) than our selection criteria, the second has a distance from the MS of 0.51 dex (above a factor of 3 ), even though it has clearly a merger-like morphology, showing a long tidal feature in the upper part. These systems can provide clues for understanding clump properties and formation also for the remaining sample.

Analyzing each starburst in more detail, in the first galaxy we can clearly see two clumps in $i$-band and four clumps in the UV, while all of them become fainter and undetected at longer wavelengths. They also occupy part of a ring surrounding the central nucleus. Simulations predict that this configuration could represent the late phase of a collision between disk galaxies (Bekki 1998), or it may be formed by tidal accretion of material from a gas rich donor galaxy (Bournaud \& Combes 2003).
Also in the second galaxy the clumps form half a ring and become brighter in the UV rest-frame. Longer wavelength images reveal that this elongated structure is connected to a single bigger system with just one main nucleus invisible in F606W and F814W filters (because highly attenuated or very old).

Finally, the last system shows multiple clumps in $i$ and $U$ band images, located below the main central nucleus visible at all those wavelengths. In particular, the brightest clump falls at the border of the main edge-on stellar disk.

Overall, the clumps shown in the above three galaxies become more prominent from redder to bluer bands (where sometimes they can outshine the nucleus itself). This would yield a higher clumpiness in the UV compared to $i$-band. On the opposite side, they become undetected in near-IR bands (even though the resolution is slightly lower). This result indicates that they are likely young structures induced by the merger rather than preexisting aggregations of older stars. Furthermore, they seem to have a low mass fraction and possibly a low SFR fraction, despite their UV prominence.

A systematic investigation of the physical properties of clumps produced in merger events, including dust attenuation, stellar ages, masses, gravitational stability, and kinematics, could be possible in the future with the availability of high spatial resolution images at multiple wavelengths for a larger subset of objects (e.g., Euclid, JWST, ERIS).

\subsection{Comparison with other studies}

As mentioned in the introduction, several other studies investigate the origin of clumpy galaxies at redshifts overlapping with our range. Puech (2010) claims that mergers may be the dominant triggering mechanisms of clumpy galaxies at $z<1$. However, his limited sample of 11 objects is representative of a very specific redshift $(\simeq 0.6)$, galaxy stellar mass $\left(\log _{10} M_{*} \sim\right.$ $\left.10.2\left[M_{\odot}\right]\right)$ and type (e.g., absence of a central bulge), which cannot be representative of all the star-forming population and of the same dynamic range spanned in our work. In addition, he found that the majority of UV clumps tend to vanish when looking at longer wavelengths, so they could be biased toward lower attenuations or higher ongoing SFRs. Also Ribeiro et al. (2017), while focusing on much higher redshifts than our work $(2<z<6)$, interpret double clumps as possible merging systems. However, if it is true, the clumps likely represent the nuclei of the two colliding galaxies, thus they should not be considered anymore as clumpy galaxies by our definition.

On the other hand, Murata et al. (2014) notice that the redshift evolution of the fraction of clumpy galaxies is inconsistent with that expected from the merger rate, thus concluding that mergers do not contribute to the population of clumpy galaxies. However, they detect clumps directly on the images without an intermediate smoothing step, which is important to facilitate the identification of high spatial frequency components and separate them from equally bright regions with smoother profiles. In addition, they identify clumpy galaxies preferentially on the main sequence. Indeed, their SFRs are derived from SED fitting (from UV to mid-IR), not allowing to select systems with obscured star-formation, which are dominant in infrared luminous mergers (Goldader et al. 2002; Calabrò et al. 2018). A similar conclusion based on the same argument is reached by Guo et al. (2015) for clumpy galaxies at $0.5<z<3$. They suggest instead that violent disk instabilities are the main triggering mechanism at high stellar masses $\left(M_{*}>10^{10.6} M_{\odot}\right)$, while minor mergers may contribute the most for intermediate mass systems with $10^{9.8}<M_{*}<10^{10.6} M_{\odot}$. However, they only consider UV 
clumps, which may disappear in the optical rest-frame, as shown by Puech (2010).

Our result should be considered as complementary to all these studies. We show that mergers are important for triggering the formation of clumps in intermediate redshift galaxies, enhancing the clumpiness at higher levels compared to other mechanisms at this cosmic epoch. However, we do not claim that all clumps are induced by mergers. Indeed, a fraction of main sequence galaxies (which are the majority $96-98 \%$ of the starforming population) with higher clumpiness are not identified as mergers and may be consistent with other formation channels, such as minor mergers or disk instabilities. Therefore, we are not in contradiction with the two previous works described above.

Finally, Lotz et al. (2004) show that local ULIRGs (which are all mergers) have an enhanced clumpiness compared to the main sequence star-forming population. Our paper thus suggests that this result can be extended at least up to redshift $\sim 1$ to starburst galaxies and to morphologically selected mergers.

\subsection{Interpreting high redshift clumpy galaxies}

We have demonstrated in previous sections that mergers can trigger the formation of stellar clumps in galaxies at $0.5<z<0.9$. Given this explanation at intermediate redshifts, we can wonder whether a similar connection also holds at earlier cosmic times.

At $z \gg 1$, young massive clumps observed in the UV and optical rest-frame, with $M_{*} \sim 10^{8} M_{\odot}$ and ages of 100-500 Myr, are generally thought to be driven by violent gravitational instabilities in gas-rich, highly turbulent primordial disk galaxies in the star-forming MS (e.g., Noguchi 1999; Immeli et al. 2004b,a; Elmegreen \& Elmegreen 2005; Elmegreen et al. 2007; Elmegreen 2008; Bournaud et al. 2007; Förster Schreiber et al. 2006, 2009). The fuel needed for star-formation and clumps triggering may be provided by relatively smooth accretion of cold gas from the cosmic web and from the CGM (Kereš et al. 2005; Dekel et al. 2009; Aumer et al. 2010). Furthermore, many studies reveal that clumpy galaxies have kinematics consistent with rotating disks (Bournaud et al. 2008; Daddi et al. 2008; Shapiro et al. 2008; Epinat et al. 2009).

Even though this scenario is physically motivated, we cannot rule out mergers as possible triggering mechanisms for the formation of clumps, in analogy to what we are showing at $z<1$. As the fraction of mergers increases monotonically at earlier times (Conselice et al. 2003), their contribution could be more important in the past, and, although they can be less efficient at rising the SFR to starburst levels (Fensch et al. 2017), our results indicate that a large clumpiness parameter can be obtained even without a strong enhancement of the star-formation activity. In addition, it is possible to preserve some degree of global rotation even during a merger event, and also a disk could rapidly reform during the latest merger stages (Rothberg \& Joseph 2006; Springel \& Hernquist 2002; Fensch et al. 2017). Therefore, many high-z systems with global rotation in the stellar or gaseous components can still be major mergers. As complementary probes to recognize these systems, we could instead look for the presence of compact, highly obscured cores in the host galaxy, which can trace late or postcoalescence merger phases (Calabrò et al. 2019; Puglisi et al. 2019).

Further clues on the origin of high-redshift clumpy galaxies come from recent ALMA observations, which show a dicotomy of clumps properties depending on the SFR level of the host galaxy. For example, Hodge et al. (2019) and Tadaki et al. (2018) observed sub-kpc clumpy structures with ALMA in the dust continuum and $\mathrm{CO}$ emission for a small subset of luminous submillimeter galaxies (SMGs) in the redshift range 1.5-5. Among the sample of eleven SMGs presented by Hodge et al. (2019), the low Sersic index profile measured in one galaxy suggests it might be a late stage merger, while interacting signatures in the optical are revealed for some of their remaining systems. They show that these structures are located in the inner $5-10 \mathrm{kpc}$ region, similarly to the distribution of our brightest clumps (in both observations and simulations), which form close to the nuclei and in the beginning of tidal tails. Additionally, the ALMA clumps produce 2 to $10 \%$ of the total galaxy emission, in agreement with the range of clumpiness that we find in the optical. This suggests that, being highly star-forming and dusty, their structures may represent still early phases of formation of clumps.

On the contrary, normal star-forming isolated disks are smoother at the same sub-mm wavelengths (e.g., Rujopakarn et al. 2016, 2019; Cibinel et al. 2017). Cibinel et al. (2017) find that UV clumps in a main sequence galaxy at redshift $=1.5$ are not visible anymore with ALMA in the $\mathrm{CO}(5-4)$ transition, which could be due to a lower gas content, or to a lower SFR of the clumps. Rujopakarn et al. (2016) studied eleven normal star-forming galaxies at redshifts $1.3-3$ with $\sim 0.4^{\prime \prime}$ resolution ALMA images. They also find no evidence of clumpy structures, which instead appear at UV wavelengths as unobscured regions, owing small SFR fractions from 0.1 to $5 \%$.

This dicotomy resembles the difference of clumpiness between IR-luminous starbursts and normal MS galaxies at $z<0.9$, and we may wonder whether it has the same physical explanation. Under the merger origin hypothesis for SMGs (e.g., Tacconi et al. 2006, 2008; Engel et al. 2010; Alaghband-Zadeh et al. 2012), it seems reasonable to think that many of the ALMA clumps observed in high- $z$ infrared luminous galaxies may be actually produced by merger interactions. However, we warn the reader that the merger origin of SMGs has not been fully assessed yet, with alternative studies claiming they are just gas rich disks representing the most massive, luminous extension of the galaxy main sequence (e.g., Davé et al. 2010; Dunlop 2011; Michałowski et al. 2012; Targett et al. 2013). Future observations with ALMA could further constrain the different hypotheses and better characterize the clumps detected in our COSMOS sample for both the MS and SB population.

\section{Summary and conclusions}

Inspired by the merger nature of infrared luminous starbursts at $0.5<z<0.9$ shown in previous works, we have studied in the same redshift range the effects of mergers on the formation of clumps by comparing HST-ACS optical rest-frame morphologies of 79 starbursts (identified in the whole COSMOS field) to a control sample of 109 normal star-forming main sequence galaxies. We have performed an additional visual merger identification among the main sequence population and applied the classical Gini- $M_{20}$ classification to select galaxies with mergerlike shapes. Our main results are summarized as follows:

First, starburst and main sequence galaxies have different clumpiness histogram distributions: the former dominate in the high clumpiness regime, while the latter are mostly found at lower clumpiness. Given the merger nature of intermediate$z$ starbursts, this suggests that mergers are likely responsible for the formation of bright clumps and for the increase of the clumpiness with respect to normal star-forming isolated disks.

Secondly, the majority (76\%) of morphological mergers (according to Gini- $M_{20}$ based criterion by Lotz et al. 2008) are 
starburst galaxies. However, $57 \%$ of starbursts are not classified as mergers from their $i$-band morphology. This suggests that the Gini- $M_{20}$ merger selection is highly incomplete, likely due to multiple effects, including the galaxy inclination, the impact parameter, the dust attenuation, and the rapid fading of merger signatures after the coalescence.

Furthermore, a larger difference among the clumpiness distributions is obtained both when comparing morphological mergers to not merger systems. In particular, Gini- $M_{20}$ mergers, regardless of their level of SFR, have a median clumpiness a factor of three higher compared to the rest of the population, and are almost entirely responsible for the high clumpiness tail observed among our sample. We also find a positive correlation between the clumpiness and the distance from the main sequence, for morphologically selected mergers.

From hydrodynamical simulations of merger galaxies with initial conditions typical for our redshift range, we also confirm that mergers can significantly enhance the clumpiness compared to isolated main sequence systems, by a similar amount to that observed in real images. Different spin-orbit couplings of colliding galaxies can fully explain the scatter of the clumpiness observed in our sample (from 0 to $20 \%$ ).

Afterward, for a sample of $21 \mathrm{SBs}$ with previous MagellanFIRE spectra, there is a mild correlation between the clumpiness and the equivalent width of Balmer and Paschen lines, suggesting a possible clumpiness evolution during the merger, decreasing from early-intermediate to later, post-coalescence stages. X-ray detected AGNs are preferentially found in low-clumpiness systems, suggesting a possible evaporation or destruction of clumps induced by AGN feedback. However, other effects (including galaxy inclination, rotation, attenuation, and impact parameter) are likely responsible for the low correlation strength $(\simeq 2 \sigma)$.

Finally, using four high-resolution images in different optical or near-IR bands, available for three clumpy starbursts identified in the COSMOS-CANDELS field, we suggest that merger induced clumps are generally young and UV-bright, likely formed during the merger rather than older preexisting stellar structures. However, a larger sample is needed to study the statistical properties of the clumps (e.g., sizes, stellar masses and ages) and investigate their evolution.

Merger triggered gas compression and fragmentation can provide the physical explanation for the formation of bright stellar clumps. We expect that this mechanism is more frequent at high redshift, given the increasing fraction of mergers at earlier epochs. This work rises a question as to the real nature of clumps observed in high redshift galaxies, suggesting that mergers could be an alternative, powerful channel for enhancing the clumpiness. If this is true, the clumpiness could be used as a complementary merger diagnostic to identify mergers from the morphology when the typical low-surface brightness interacting features (e.g., tidal tails) become too faint.

Deeper images in the optical and near-IR rest-frame with Euclid ERIS and JWST will allow in the near future the detection of clumps and their physical characterization (through a multiwavelength approach) for larger statistical samples of highredshift clumpy galaxies, with similar spatial resolutions to those considered here. In addition, they will facilitate the study of their environment and the morphological or dynamical properties of their host galaxies.

Acknowledgements. We thank the anonymous referee for comments improving the quality of this manuscript. I.D. is supported by the European Union's Horizon 2020 research and innovation program under the Marie SkłodowskaCurie grant agreement No 788679. S.J. acknowledges financial support from the
Spanish Ministry of Science, Innovation and Universities (MICIU) under grant AYA2017-84061-P, co-financed by FEDER (European Regional Development Funds).

\section{References}

Alaghband-Zadeh, S., Chapman, S. C., Swinbank, A. M., et al. 2012, MNRAS, 424, 2232

Alonso-Herrero, A., Rieke, G. H., Rieke, M. J., et al. 2006, ApJ, 650, 835

Aumer, M., Burkert, A., Johansson, P. H., \& Genzel, R. 2010, ApJ, 719, 1230

Bekki, K. 1998, ApJ, 499, 635

Bournaud, F. 2016, Galact. Bulges, 418, 355

Bournaud, F., \& Combes, F. 2003, A\&A, 401, 817

Bournaud, F., Elmegreen, B. G., \& Elmegreen, D. M. 2007, ApJ, 670, 237

Bournaud, F., Daddi, E., Elmegreen, B. G., et al. 2008, A\&A, 486, 741

Bournaud, F., Powell, L. C., Chapon, D., \& Teyssier, R. 2011a, Astrophysical

Dynamics: From Stars to Galaxies, 271, 160

Bournaud, F., Dekel, A., Teyssier, R., et al. 2011b, ApJ, 741, L33

Bournaud, F., Juneau, S., Le Floc'h, E., et al. 2012, ApJ, 757, 81

Bournaud, F., Perret, V., Renaud, F., et al. 2014, ApJ, 780, 57

Bruzual, G., \& Charlot, S. 2003, MNRAS, 344, 1000

Cacciato, M., Dekel, A., \& Genel, S. 2012, MNRAS, 421, 818

Calabrò, A., Daddi, E., Cassata, P., et al. 2018, ApJ, 862, L22

Calabrò, A., Daddi, E., Puglisi, A., et al. 2019, A\&A, 623, A64

Calzetti, D., Armus, L., Bohlin, R. C., et al. 2000, ApJ, 533, 682

Ceverino, D., Dekel, A., \& Bournaud, F. 2010, MNRAS, 404, 2151

Chabrier, G. 2003, PASP, 115, 763

Chapman, S. C., Windhorst, R., Odewahn, S., Yan, H., \& Conselice, C. 2003 ApJ, 599, 92

Cibinel, A., Daddi, E., Bournaud, F., et al. 2017, MNRAS, 469, 4683

Cibinel, A., Daddi, E., Sargent, M. T., et al. 2019, MNRAS, 485, 5631

Clements, D. L., Sutherland, W. J., McMahon, R. G., \& Saunders, W. 1996, MNRAS, 279, 477

Cochrane, R. K., Hayward, C. C., Anglés-Alcázar, D., et al. 2019, MNRAS, 488, 1779

Combes, F., García-Burillo, S., Braine, J., et al. 2013, A\&A, 550, A41

Conselice, C. J., Bershady, M. A., Dickinson, M., \& Papovich, C. 2003, AJ, 126, 1183

Cox, T. J., Jonsson, P., Primack, J. R., \& Somerville, R. S. 2006, MNRAS, 373, 1013

Daddi, E., Dickinson, M., Morrison, G., et al. 2007, ApJ, 670, 156

Daddi, E., Dannerbauer, H., Elbaz, D., et al. 2008, ApJ, 673, L21

Daddi, E., Bournaud, F., Walter, F., et al. 2010, ApJ, 713, 686

Davé, R., Finlator, K., Oppenheimer, B. D., et al. 2010, MNRAS, 404, 1355

Dekel, A., \& Birnboim, Y. 2006, MNRAS, 368, 2

Dekel, A., Sari, R., \& Ceverino, D. 2009, ApJ, 703, 785

Delgado-Serrano, R., Hammer, F., Yang, Y. B., et al. 2010, A\&A, 509, A78

Di Matteo, P., Bournaud, F., Martig, M., et al. 2008, A\&A, 492, 31

Draine, B. T., \& Li, A. 2007, ApJ, 657, 810

Dubois, Y., \& Teyssier, R. 2008, Pathways Through an Eclectic Universe, 390, 388

Duc, P. A., \& Renaud, F. 2013, Lecture Notes in Physics (Berlin: Springer Verlag), 861, 327

Dunlop, J. S. 2011, Galaxy Evolution: Infrared to Millimeter Wavelength Perspective (San Francisco: Astronomical Society of the Pacific), 209

Elbaz, D., \& Cesarsky, C. J. 2003, Science, 300, 270

Elbaz, D., Daddi, E., Le Borgne, D., et al. 2007, A\&A, 468, 33

Elmegreen, B. G. 2008, Mass Loss from Stars and the Evolution of Stellar Clusters (San Francisco: Astronomical Society of the Pacific), 388, 249

Elmegreen, B. G., \& Elmegreen, D. M. 2005, ApJ, 627, 632

Elmegreen, D. M., Elmegreen, B. G., Rubin, D. S., \& Schaffer, M. A. 2005, ApJ, 631,85

Elmegreen, D. M., Elmegreen, B. G., Ferguson, T., \& Mullan, B. 2007, ApJ, 663, 734

Elmegreen, B. G., Bournaud, F., \& Elmegreen, D. M. 2008, ApJ, 688, 67

Elmegreen, B. G., Elmegreen, D. M., Sánchez Almeida, J., et al. 2013, ApJ, 774, 86

Engel, H., Tacconi, L. J., Davies, R. I., et al. 2010, ApJ, 724, 233

Epinat, B., Contini, T., Le Fèvre, O., et al. 2009, A\&A, 504, 789

Fensch, J., Renaud, F., Bournaud, F., et al. 2017, MNRAS, 465, 1934

Förster Schreiber, N. M., Genzel, R., Lehnert, M. D., et al. 2006, ApJ, 645, 1062

Förster Schreiber, N. M., Genzel, R., Bouché, N., et al. 2009, ApJ, 706, 1364

Freundlich, J., Combes, F., Tacconi, L. J., et al. 2019, A\&A, 622, A105

García-Marín, M., Colina, L., \& Arribas, S. 2009, A\&A, 505, 1017

Garland, C. A., Pisano, D. J., Mac Low, M.-M., et al. 2015, ApJ, 807, 134

Glasser, G. J. 1962, J. Am. Stat. Assoc., 57, 648

Goldader, J. D., Meurer, G., Heckman, T. M., et al. 2002, ApJ, 568, 651 
Governato, F., Brook, C., Mayer, L., et al. 2010, Nature, 463, 203 Guo, Y., Ferguson, H. C., Bell, E. F., et al. 2015, ApJ, 800, 39 Hayward, C. C., Kereš, D., Jonsson, P., et al. 2011, ApJ, 743, 159 Hodge, J. A., Smail, I., Walter, F., et al. 2019, ApJ, 876, 130 Jin, S., Daddi, E., Liu, D., et al. 2018, ApJ, 864, 56 Jonsson, P., Groves, B. A., \& Cox, T. J. 2010, MNRAS, 403, 17 Immeli, A., Samland, M., Gerhard, O., \& Westera, P. 2004a, A\&A, 413, 547 Immeli, A., Samland, M., Westera, P., \& Gerhard, O. 2004b, ApJ, 611, 20 Kereš, D., Katz, N., Weinberg, D. H., \& Davé, R. 2005, MNRAS, 363, 2 Koekemoer, A. M., Aussel, H., Calzetti, D., et al. 2007, ApJS, 172, 196

Koekemoer, A. M., Faber, S. M., Ferguson, H. C., et al. 2011, ApJS, 197, 36 Kraljic, K., Bournaud, F., \& Martig, M. 2012, ApJ, 757, 60

Laigle, C., McCracken, H. J., Ilbert, O., et al. 2016, ApJS, 224, 24

Law, D. R., Steidel, C. C., Erb, D. K., et al. 2009, ApJ, 697, 2057

Lotz, J. M., Primack, J., \& Madau, P. 2004, AJ, 128, 163

Lotz, J. M., Jonsson, P., Cox, T. J., \& Primack, J. R. 2008, MNRAS, 391, 1137

Madau, P., \& Dickinson, M. 2014, ARA\&A, 52, 415

Matsui, H., Tanikawa, A., \& Saitoh, T. R. 2019, PASJ, 71, 19

Michałowski, M. J., Dunlop, J. S., Cirasuolo, M., et al. 2012, A\&A, 541, A85

Moody, C. E., Guo, Y., Mandelker, N., et al. 2014, MNRAS, 444, 1389

Mullaney, J. R., Alexander, D. M., Goulding, A. D., \& Hickox, R. C. 2011, MNRAS, 414, 1082

Murata, K. L., Kajisawa, M., Taniguchi, Y., et al. 2014, ApJ, 786, 15

Nelson, E. J., van Dokkum, P. G., Momcheva, I. G., et al. 2016, ApJ, 817, L9

Nevin, R., Blecha, L., Comerford, J., \& Greene, J. 2019, ApJ, 872, 76

Noeske, K. G., Weiner, B. J., Faber, S. M., et al. 2007, ApJ, 660, L43

Noguchi, M. 1999, ApJ, 514, 77

Piqueras López, J., Colina, L., Arribas, S., et al. 2016, A\&A, 590, A67

Puech, M. 2010, MNRAS, 406, 535

Puglisi, A., Daddi, E., Liu, D., et al. 2019, ApJ, 877, L23

Renaud, F., Bournaud, F., Kraljic, K., \& Duc, P.-A. 2014, MNRAS, 442, L33

Ribeiro, B., Le Fèvre, O., Cassata, P., et al. 2017, A\&A, 608, A16
Rodrigues, M., Puech, M., Hammer, F., Rothberg, B., \& Flores, H. 2012, MNRAS, 421, 2888

Rodriguez-Gomez, V., Pillepich, A., Sales, L. V., et al. 2016, MNRAS, 458, 2371 Rodriguez-Gomez, V., Snyder, G. F., Lotz, J. M., et al. 2019, MNRAS, 483, 4140 Rothberg, B., \& Joseph, R. D. 2006, AJ, 132, 976

Rujopakarn, W., Dunlop, J. S., Rieke, G. H., et al. 2016, ApJ, 833, 12

Rujopakarn, W., Daddi, E., Rieke, G. H., et al. 2019, ApJ, 882, 107

Salmi, F., Daddi, E., Elbaz, D., et al. 2012, ApJ, 754, L14

Rodighiero, G., Daddi, E., Baronchelli, I., et al. 2011, ApJ, 739, L40

Sanders, D. B., \& Mirabel, I. F. 1996, ARA\&A, 34, 749

Sargent, M. T., Daddi, E., Béthermin, M., et al. 2014, ApJ, 793, 19

Schreiber, C., Pannella, M., Elbaz, D., et al. 2015, A\&A, 575, A74

Shapiro, K. L., Genzel, R., Förster Schreiber, N. M., et al. 2008, ApJ, 682, 231

Shapiro, K. L., Genzel, R., \& Förster Schreiber, N. M. 2010, MNRAS, 403, L36 Sheth, K., Melbourne, J., Elmegreen, D. M., et al. 2012, ApJ, 758, 136

Schmidt, M. 1959, ApJ, 129, 243

Scoville, N. Z., Yun, M. S., \& Bryant, P. M. 1997, ApJ, 484, 702

Scoville, N., Sheth, K., Aussel, H., et al. 2016, ApJ, 820, 83

Silverman, J. D., Daddi, E., Rodighiero, G., et al. 2015, ApJ, 812, L23

Somerville, R. S. 2001, Galaxy Disks and Disk Galaxies (San Francisco: Astronomical Society of the Pacific), 230, 477

Sparre, M., \& Springel, V. 2016, MNRAS, 462, 2418

Springel, V., \& Hernquist, L. 2002, MNRAS, 333, 649

Tacconi, L. J., Neri, R., Chapman, S. C., et al. 2006, ApJ, 640, 228

Tacconi, L. J., Genzel, R., Smail, I., et al. 2008, ApJ, 680, 246

Tacconi, L. J., Genzel, R., Neri, R., et al. 2010, Nature, 463, 781

Tadaki, K., Iono, D., Yun, M. S., et al. 2018, Nature, 560, 613

Targett, T. A., Dunlop, J. S., Cirasuolo, M., et al. 2013, MNRAS, 432, 2012 Teyssier, R. 2002, A\&A, 385, 337

Teyssier, R., Chapon, D., \& Bournaud, F. 2010, ApJ, 720, L149

Wuyts, S., Förster Schreiber, N. M., Genzel, R., et al. 2012, ApJ, 753, 114

Zanella, A., Daddi, E., Le Floc'h, E., et al. 2015, Nature, 521, 54

Zanella, A., Le Floc'h, E., Harrison, C. M., et al. 2019, MNRAS, 489, 2792 


\section{Appendix A: Comparison between the clumpiness and other quantities}

In this appendix we show how the clumpiness compares with other parameters considered in the main part of the paper. Figure A.1 displays the clumpiness as a function of the $i$-band magnitude and the elongation of our targets. This explains the final sample selection made for our analysis (described in Sects. 2.6 and 3), highlighted by the gray vertical lines. In Fig. A.2 we compare the clumpiness to different morphological indicators, that are, the Gini coefficient, the $M_{20}$ parameter and the mergerness. The first two diagrams show the lack of correlation between the two quantities in the $x$ and $y$-axis. Finally, Fig. A.3-top shows the redshift distribution of our sample, ranging $0.5<z<0.9$ and with a median $z$ of 0.73 . Figures A.3bottom and A.4 demonstrate, respectively, that the clumpiness does not depend on the stellar mass, and does not significantly evolve with redshift (within our uncertainties).
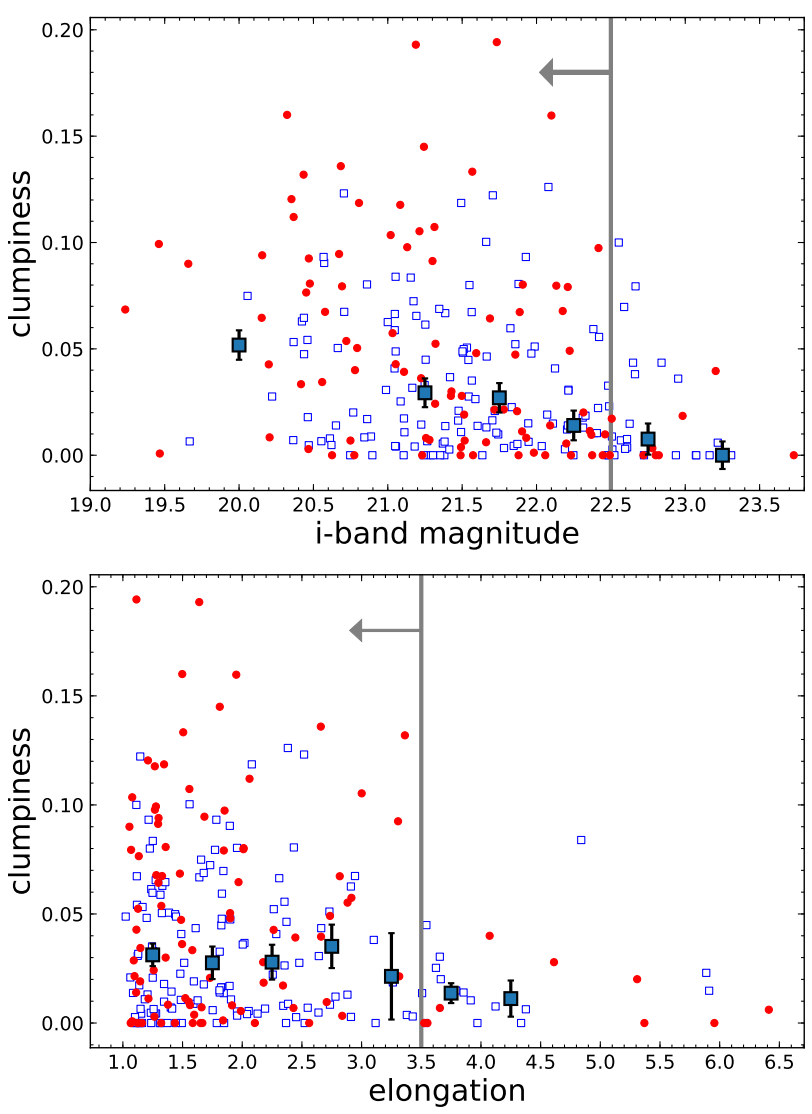

Fig. A.1. Top: clumpiness vs $i$-band total magnitude $\left(i_{\mathrm{mag}}\right)$ for our initial sample of 96 starburst and 145 main sequence galaxies (with red filled circles and blue empty squares, respectively). The median clumpiness values and errors (gray squares with black error bars) are derived for six bins of $i_{\text {mag }}$. Our selection cut $\left(i_{\text {mag }}<22.5\right)$ is highlighted with a vertical gray line. Bottom: clumpiness vs elongation for the same sample as above, with median clumpiness and errors estimated in seven bins of elongation. The vertical gray line indicates our cut for the final selection (elongation $<3.5$ ), even though we remember that one additional nearly edge-on MS galaxy was also removed by visual inspection. 

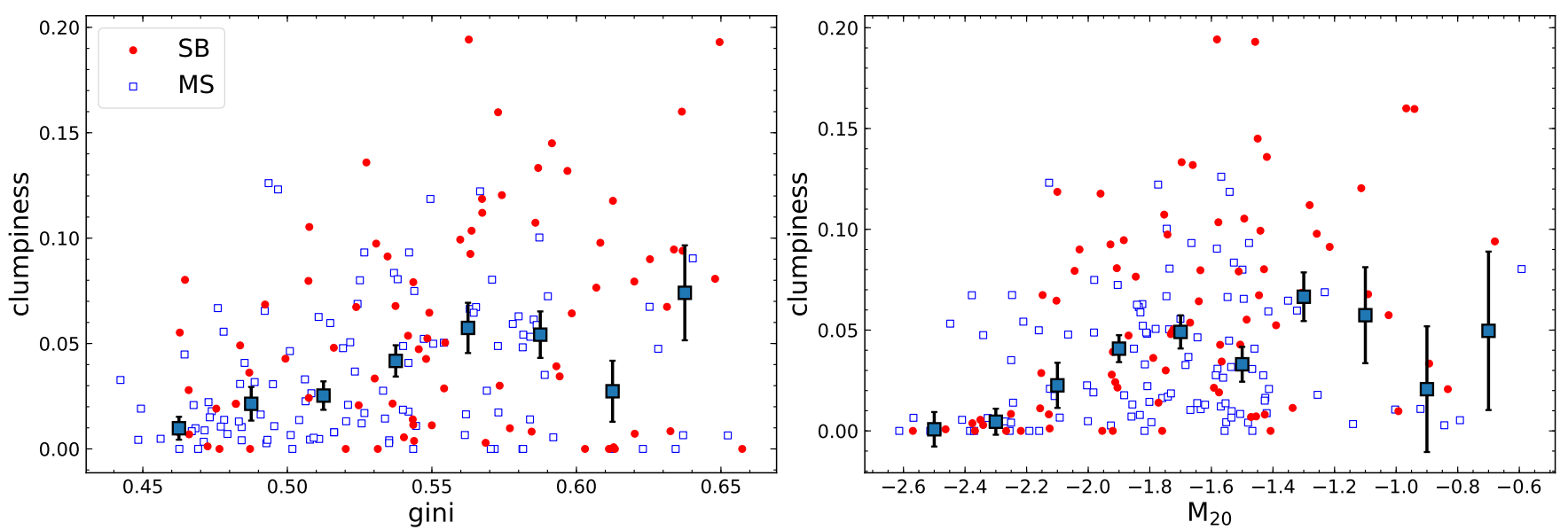

Fig. A.2. Diagrams comparing the clumpiness of our final galaxy sample to the Gini and $M_{20}$ coefficients (first and second panels, respectively), and to the mergerness parameter, defined in Eq. (3). Starbursts are shown with red filled circles, while main sequence galaxies are empty squares and color coded in blue. The median clumpiness (and errors), computed in bins of Gini, $M_{20}$ and mergerness, are represented with gray squares and black error bars.
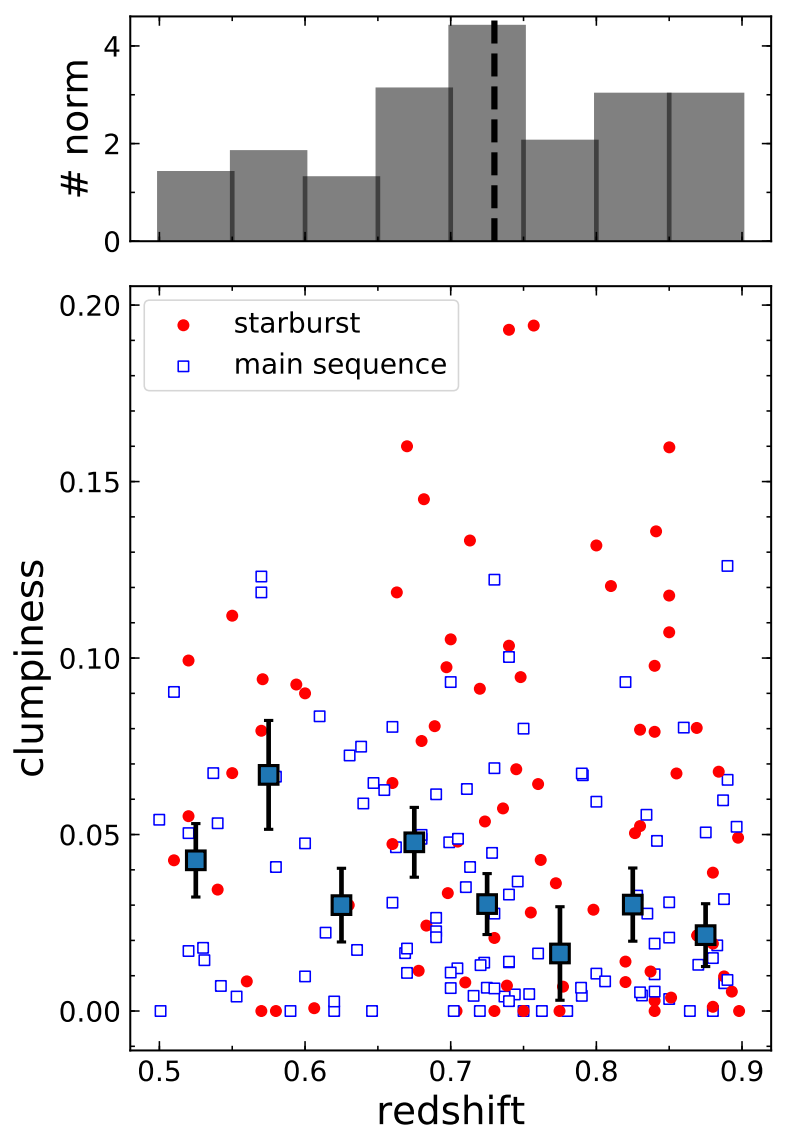

Fig. A.3. Redshift vs clumpiness for our final selected galaxies, with gray squares and black error bars representing the median clumpiness and error calculated inside eight redshift bins. Top: redshift histogram distribution of our galaxies, with the median redshift $\left(z_{\text {med }}=0.73\right)$ highlighted with a black dashed line.

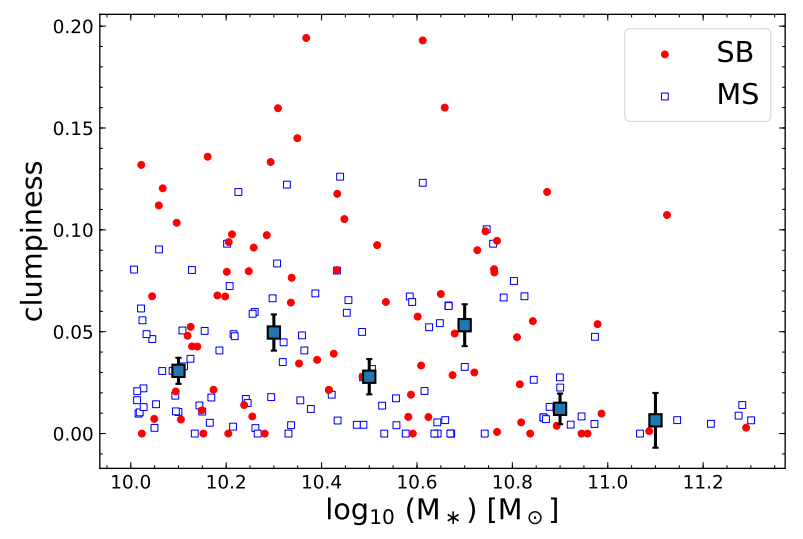

Fig. A.4. Stellar mass vs clumpiness for our final sample of SB and MS galaxies (with the same color coding and markers of Fig. A.2), with median clumpiness (and error) in six $M_{*}$ bins superimposed with gray squares. 
Table A.1. Main physical and morphological properties of galaxies analyzed in this paper.

\begin{tabular}{|c|c|c|c|c|c|c|c|c|}
\hline $\begin{array}{l}\text { ID } \\
(1)\end{array}$ & $\begin{array}{l}\mathrm{RA} \\
(2)\end{array}$ & $\begin{array}{l}\text { Dec } \\
(3)\end{array}$ & $\begin{array}{l}\text { Redshift } \\
(4)\end{array}$ & $\begin{array}{l}c^{\prime} \\
(5)\end{array}$ & $\begin{array}{l}\text { Clumpiness } \\
(6)\end{array}$ & $\begin{array}{l}\text { Mergerness } \\
(7)\end{array}$ & $\begin{array}{l}M_{*}\left[M_{\odot}\right] \\
(8)\end{array}$ & $\begin{array}{l}\mathrm{SFR}_{\mathrm{IR}}\left[M_{\odot} \mathrm{yr}^{-1}\right] \\
(9)\end{array}$ \\
\hline 217536 & 149.524108 & 1.606315 & $0.87 \pm 0.03$ & 0.02 & 0.02 & 0.07 & 10.4 & $74.25 \pm 6.633$ \\
\hline 220514 & 149.58302 & 1.61253 & $0.89 \pm 0.03$ & 0.01 & 0.01 & 0.07 & 10.9 & $49.96 \pm 4.284$ \\
\hline 222723 & 150.173213 & 1.616315 & $0.61 \pm 0.01$ & 0.0 & 0.0 & 0.06 & 10.8 & $151.2 \pm 6.357$ \\
\hline 222977 & 150.414727 & 1.618189 & $0.75 \pm 0.0$ & 0.03 & 0.04 & 0.04 & 10.1 & $15.29 \pm 2.6$ \\
\hline 223715 & 149.765371 & 1.617017 & $0.52 \pm 0.0$ & 0.09 & 0.1 & -0.03 & 10.7 & $52.4 \pm 0.805$ \\
\hline 228149 & 149.816844 & 1.627891 & $0.63 \pm 0.12$ & 0.06 & 0.07 & 0.01 & 10.2 & $14.57 \pm 6.078$ \\
\hline 239406 & 150.338269 & 1.644141 & $0.73 \pm 0.02$ & 0.04 & 0.04 & 0.08 & 10.3 & $12.82 \pm 1.733$ \\
\hline 244932 & 149.460788 & 1.651282 & $0.72 \pm 0.02$ & 0.01 & 0.01 & 0.06 & 10.7 & $19.61 \pm 2.242$ \\
\hline 245158 & 150.188543 & 1.654977 & $0.52 \pm 0.0$ & 0.05 & 0.06 & 0.07 & 10.8 & $83.05 \pm 1.227$ \\
\hline 247210 & 150.487543 & 1.656873 & $0.83 \pm 0.0$ & 0.05 & 0.05 & -0.02 & 10.1 & $41.72 \pm 1.106$ \\
\hline 249989 & 150.685402 & 1.661076 & $0.74 \pm 0.04$ & 0.06 & 0.06 & -0.09 & 10.6 & $102.4 \pm 14.72$ \\
\hline 259328 & 149.916036 & 1.676354 & $0.74 \pm 0.0$ & 0.03 & 0.03 & 0.08 & 10.1 & $26.09 \pm 0.57$ \\
\hline 271645 & 149.607229 & 1.694341 & $0.74 \pm 0.08$ & 0.0 & 0.0 & 0.14 & 11.0 & $15.24 \pm 3.196$ \\
\hline 275629 & 150.182349 & 1.700807 & $0.74 \pm 0.0$ & 0.14 & 0.19 & -0.11 & 10.6 & $101.3 \pm 0.924$ \\
\hline 280708 & 149.969049 & 1.710048 & $0.86 \pm 0.0$ & 0.09 & 0.1 & 0.02 & 10.1 & $36.63 \pm 4.083$ \\
\hline 286337 & 149.808093 & 1.717684 & $0.75 \pm 0.0$ & 0.0 & 0.0 & 0.08 & 10.6 & $17.23 \pm 1.73$ \\
\hline 287281 & 149.662106 & 1.719228 & $0.71 \pm 0.02$ & 0.06 & 0.06 & 0.01 & 10.7 & $44.15 \pm 5.46$ \\
\hline 289993 & 149.951404 & 1.725684 & $0.68 \pm 0.0$ & 0.01 & 0.01 & -0.03 & 10.1 & $42.29 \pm 0.49$ \\
\hline 293450 & 149.496283 & 1.729198 & $0.86 \pm 0.06$ & 0.06 & 0.07 & 0.01 & 10.2 & $45.48 \pm 7.794$ \\
\hline 294096 & 149.51561 & 1.731195 & $0.67 \pm 0.06$ & 0.01 & 0.02 & -0.03 & 10.0 & $4.835 \pm 1.635$ \\
\hline 295213 & 150.421274 & 1.731761 & $0.64 \pm 0.04$ & 0.07 & 0.07 & 0.06 & 10.8 & $47.17 \pm 6.101$ \\
\hline 297678 & 149.582631 & 1.738114 & $0.87 \pm 0.03$ & 0.07 & 0.08 & 0.06 & 10.4 & $78.84 \pm 5.764$ \\
\hline 300643 & 149.779375 & 1.741341 & $0.53 \pm 0.0$ & 0.02 & 0.02 & 0.03 & 10.3 & $5.695 \pm 1.548$ \\
\hline 308894 & 150.259527 & 1.756639 & $0.88 \pm 0.0$ & 0.04 & 0.05 & 0.06 & 10.1 & $25.86 \pm 3.1$ \\
\hline 314674 & 149.922125 & 1.764706 & $0.89 \pm 0.01$ & 0.06 & 0.07 & 0.05 & 10.5 & $22.89 \pm 3.078$ \\
\hline 319784 & 150.073982 & 1.772639 & $0.73 \pm 0.0$ & 0.02 & 0.02 & -0.08 & 10.1 & $47.72 \pm 0.947$ \\
\hline 326384 & 149.517858 & 1.783572 & $0.71 \pm 0.04$ & 0.1 & 0.13 & -0.02 & 10.3 & $125.0 \pm 14.8$ \\
\hline 327954 & 149.493504 & 1.786618 & $0.61 \pm 0.11$ & 0.02 & 0.02 & 0.11 & 10.0 & $9.337 \pm 4.839$ \\
\hline 331390 & 150.640087 & 1.78974 & $0.67 \pm 0.0$ & 0.01 & 0.01 & 0.06 & 10.1 & $10.15 \pm 2.1$ \\
\hline 331485 & 149.494555 & 1.791539 & $0.71 \pm 0.02$ & 0.02 & 0.04 & 0.06 & 10.3 & $15.02 \pm 2.365$ \\
\hline 332156 & 150.23588 & 1.791352 & $0.83 \pm 0.16$ & 0.0 & 0.0 & 0.14 & 10.9 & $29.63 \pm 11.85$ \\
\hline 340778 & 150.458433 & 1.803992 & $0.75 \pm 0.0$ & 0.0 & 0.0 & 0.15 & 11.2 & $39.87 \pm 1.179$ \\
\hline 350404 & 150.20613 & 1.822038 & $0.74 \pm 0.0$ & 0.0 & 0.0 & 0.05 & 10.3 & $14.75 \pm 4.196$ \\
\hline 358661 & 149.710063 & 1.832255 & $0.5 \pm 0.13$ & 0.0 & 0.0 & 0.12 & 10.3 & $6.222 \pm 3.722$ \\
\hline 366376 & 150.138331 & 1.844028 & $0.57 \pm 0.0$ & 0.08 & 0.09 & -0.21 & 10.2 & $38.12 \pm 0.414$ \\
\hline 368717 & 149.718723 & 1.849718 & $0.67 \pm 0.0$ & 0.14 & 0.16 & -0.17 & 10.7 & $67.19 \pm 0.758$ \\
\hline 371578 & 150.674055 & 1.85337 & $0.51 \pm 0.0$ & 0.04 & 0.04 & 0.0 & 10.1 & $26.9 \pm 0.222$ \\
\hline 371675 & 149.901208 & 1.85448 & $0.74 \pm 0.0$ & 0.01 & 0.01 & 0.08 & 10.1 & $8.38 \pm 2.119$ \\
\hline 371886 & 149.900477 & 1.854753 & $0.64 \pm 0.0$ & 0.01 & 0.02 & 0.05 & 10.6 & $7.781 \pm 1.071$ \\
\hline 372591 & 150.503808 & 1.854601 & $0.75 \pm 0.0$ & 0.07 & 0.08 & 0.01 & 10.4 & $36.58 \pm 1.03$ \\
\hline 387242 & 150.222431 & 1.878024 & $0.84 \pm 0.0$ & 0.08 & 0.1 & -0.1 & 10.2 & $51.69 \pm 1.204$ \\
\hline 387454 & 150.652196 & 1.877237 & $0.59 \pm 0.0$ & 0.08 & 0.09 & 0.04 & 10.5 & $64.74 \pm 1.105$ \\
\hline 387747 & 149.916015 & 1.879961 & $0.84 \pm 0.0$ & 0.07 & 0.08 & -0.0 & 10.8 & $102.4 \pm 0.974$ \\
\hline 400118 & 150.051501 & 1.898621 & $0.57 \pm 0.0$ & 0.1 & 0.12 & -0.0 & 10.2 & $11.69 \pm 1.625$ \\
\hline 402258 & 150.648684 & 1.902372 & $0.74 \pm 0.0$ & 0.09 & 0.1 & -0.01 & 10.1 & $42.46 \pm 0.332$ \\
\hline 409602 & 149.578964 & 1.913372 & $0.83 \pm 0.0$ & 0.07 & 0.08 & 0.05 & 10.2 & $52.3 \pm 0.685$ \\
\hline 409814 & 150.198597 & 1.914859 & $0.87 \pm 0.0$ & 0.02 & 0.02 & 0.06 & 10.2 & $46.7 \pm 0.862$ \\
\hline 412250 & 150.741711 & 1.91764 & $0.68 \pm 0.03$ & 0.1 & 0.14 & -0.06 & 10.3 & $109.4 \pm 10.24$ \\
\hline 416314 & 150.663036 & 1.924054 & $0.83 \pm 0.0$ & 0.0 & 0.01 & -0.07 & 10.2 & $12.06 \pm 0.433$ \\
\hline 418804 & 149.935142 & 1.927629 & $0.68 \pm 0.0$ & 0.07 & 0.08 & -0.02 & 10.3 & $47.2 \pm 0.721$ \\
\hline 431551 & 149.568139 & 1.94866 & $0.54 \pm 0.02$ & 0.01 & 0.01 & 0.11 & 10.9 & $13.7 \pm 1.814$ \\
\hline
\end{tabular}

Notes. Table columns: (1,2,3) Identification number, RA and Dec (in degree) from Laigle et al. (2016); (4) photometric redshift (or spectroscopic if available) from Jin et al. (2018) ; (5,6) clumpiness parameters (clumpiness is estimated as in Eq. (4)), while in $c^{\prime}$ the flux of the clumps is divided by the total flux of the galaxy (including the nuclei) inside the segmentation map; (7) mergerness parameter, estimated as in Eq. (3); (8) stellar mass from Laigle et al. (2016), with typical uncertainty of 0.1 dex; (9) $S_{F R}$ from Jin et al. (2018). 
Table A.1. continued.

\begin{tabular}{|c|c|c|c|c|c|c|c|c|}
\hline $\begin{array}{l}\text { ID } \\
(1)\end{array}$ & $\begin{array}{l}\text { RA } \\
(2)\end{array}$ & $\begin{array}{l}\text { Dec } \\
(3)\end{array}$ & $\begin{array}{l}\text { Redshift } \\
(4)\end{array}$ & $\begin{array}{l}c^{\prime} \\
(5)\end{array}$ & $\begin{array}{l}\text { Clumpiness } \\
(6)\end{array}$ & $\begin{array}{l}\text { Mergerness } \\
(7)\end{array}$ & $\begin{array}{l}M_{*}\left[M_{\odot}\right] \\
(8)\end{array}$ & $\begin{array}{l}\mathrm{SFR}_{\mathrm{IR}}\left[M_{\odot} \mathrm{yr}^{-1}\right] \\
(9)\end{array}$ \\
\hline 431596 & 149.948236 & 1.950357 & $0.85 \pm 0.0$ & 0.03 & 0.03 & 0.07 & 10.1 & $15.5 \pm 0.389$ \\
\hline 436769 & 150.617708 & 1.958166 & $0.65 \pm 0.08$ & 0.0 & 0.0 & 0.01 & 10.5 & $14.6 \pm 5.141$ \\
\hline 439419 & 150.301785 & 1.962747 & $0.7 \pm 0.02$ & 0.01 & 0.01 & 0.02 & 10.4 & $15.06 \pm 1.283$ \\
\hline 444878 & 150.687694 & 1.970867 & $0.76 \pm 0.12$ & 0.16 & 0.19 & -0.01 & 10.4 & $85.03 \pm 27.02$ \\
\hline 447374 & 150.543608 & 1.974443 & $0.79 \pm 0.03$ & 0.06 & 0.07 & 0.1 & 10.8 & $26.29 \pm 3.279$ \\
\hline 451272 & 150.250512 & 1.980928 & $0.72 \pm 0.0$ & 0.08 & 0.09 & -0.03 & 10.3 & $45.3 \pm 0.57$ \\
\hline 451426 & 149.651824 & 1.981124 & $0.79 \pm 0.0$ & 0.0 & 0.0 & 0.1 & 10.5 & $14.85 \pm 3.362$ \\
\hline 466112 & 149.999276 & 2.005995 & $0.76 \pm 0.0$ & 0.05 & 0.06 & -0.04 & 10.3 & $144.0 \pm 1.052$ \\
\hline 472775 & 150.481476 & 2.013621 & $0.68 \pm 0.02$ & 0.02 & 0.02 & 0.09 & 10.8 & $159.7 \pm 11.82$ \\
\hline 473147 & 150.399682 & 2.014254 & $0.72 \pm 0.0$ & 0.05 & 0.05 & 0.02 & 11.0 & $107.1 \pm 1.269$ \\
\hline 474838 & 149.543724 & 2.01889 & $0.77 \pm 0.02$ & 0.03 & 0.04 & 0.09 & 10.4 & $58.87 \pm 3.831$ \\
\hline 475972 & 149.526013 & 2.020502 & $0.62 \pm 0.0$ & 0.0 & 0.0 & 0.05 & 10.7 & $8.241 \pm 2.693$ \\
\hline 478933 & 150.666347 & 2.025354 & $0.8 \pm 0.0$ & 0.13 & 0.13 & -0.03 & 10.0 & $53.94 \pm 0.785$ \\
\hline 480125 & 149.749497 & 2.027741 & $0.7 \pm 0.0$ & 0.0 & 0.01 & 0.05 & 11.3 & $31.46 \pm 1.037$ \\
\hline 486542 & 150.761399 & 2.040174 & $0.88 \pm 0.0$ & 0.06 & 0.07 & -0.05 & 10.2 & $103.9 \pm 2.043$ \\
\hline 488940 & 149.831475 & 2.040881 & $0.53 \pm 0.13$ & 0.01 & 0.01 & 0.05 & 10.1 & $4.905 \pm 2.899$ \\
\hline 493881 & 150.749674 & 2.047066 & $0.6 \pm 0.0$ & 0.08 & 0.09 & -0.01 & 10.7 & $129.2 \pm 1.006$ \\
\hline 500548 & 149.62879 & 2.05949 & $0.63 \pm 0.0$ & 0.02 & 0.03 & 0.0 & 10.7 & $67.56 \pm 1.033$ \\
\hline 503971 & 150.65256 & 2.063559 & $0.6 \pm 0.0$ & 0.04 & 0.05 & 0.03 & 11.0 & $9.35 \pm 1.21$ \\
\hline 505311 & 150.089164 & 2.065345 & $0.73 \pm 0.0$ & 0.03 & 0.03 & -0.02 & 10.9 & $34.49 \pm 3.37$ \\
\hline 506131 & 149.528615 & 2.0665 & $0.57 \pm 0.0$ & 0.06 & 0.08 & -0.0 & 10.2 & $39.06 \pm 0.442$ \\
\hline 508753 & 149.928339 & 2.07214 & $0.68 \pm 0.0$ & 0.04 & 0.05 & 0.03 & 10.2 & $23.69 \pm 0.944$ \\
\hline 508907 & 149.856873 & 2.072084 & $0.69 \pm 0.0$ & 0.05 & 0.06 & 0.0 & 10.0 & $15.84 \pm 1.112$ \\
\hline 515278 & 150.523885 & 2.082297 & $0.89 \pm 0.0$ & 0.11 & 0.13 & 0.06 & 10.4 & $28.73 \pm 3.716$ \\
\hline 516551 & 150.205761 & 2.083436 & $0.78 \pm 0.01$ & 0.01 & 0.01 & 0.07 & 10.1 & $44.4 \pm 2.191$ \\
\hline 518855 & 150.12551 & 2.08703 & $0.89 \pm 0.0$ & 0.0 & 0.01 & 0.12 & 10.8 & $142.3 \pm 1.436$ \\
\hline 519651 & 150.430196 & 2.086883 & $0.66 \pm 0.0$ & 0.05 & 0.06 & 0.07 & 10.5 & $137.1 \pm 1.312$ \\
\hline 529521 & 150.103938 & 2.104858 & $0.83 \pm 0.0$ & 0.03 & 0.03 & 0.12 & 10.7 & $15.19 \pm 3.556$ \\
\hline 532321 & 149.893588 & 2.107745 & $0.74 \pm 0.07$ & 0.01 & 0.01 & -0.09 & 10.0 & $52.17 \pm 10.76$ \\
\hline 536590 & 149.918602 & 2.113077 & $0.72 \pm 0.06$ & 0.01 & 0.01 & 0.04 & 10.5 & $10.65 \pm 3.274$ \\
\hline 539760 & 149.880566 & 2.120673 & $0.68 \pm 0.0$ & 0.04 & 0.05 & 0.08 & 10.5 & $15.08 \pm 1.726$ \\
\hline 544522 & 150.052099 & 2.126677 & $0.66 \pm 0.0$ & 0.1 & 0.12 & 0.06 & 10.9 & $90.06 \pm 0.794$ \\
\hline 545104 & 150.451931 & 2.127888 & $0.84 \pm 0.0$ & 0.0 & 0.0 & 0.12 & 10.3 & $85.6 \pm 1.039$ \\
\hline 545185 & 149.528016 & 2.12725 & $0.54 \pm 0.0$ & 0.03 & 0.03 & -0.04 & 10.4 & $127.3 \pm 1.155$ \\
\hline 546483 & 150.674581 & 2.131899 & $0.9 \pm 0.0$ & 0.0 & 0.0 & 0.07 & 10.0 & $39.28 \pm 1.527$ \\
\hline 562400 & 150.512919 & 2.152049 & $0.56 \pm 0.0$ & 0.01 & 0.01 & 0.01 & 10.3 & $40.5 \pm 0.553$ \\
\hline 571040 & 149.509664 & 2.167659 & $0.84 \pm 0.0$ & 0.02 & 0.02 & 0.16 & 10.4 & $18.17 \pm 1.262$ \\
\hline 574334 & 150.346743 & 2.170359 & $0.85 \pm 0.0$ & 0.1 & 0.12 & -0.01 & 10.4 & $132.5 \pm 1.197$ \\
\hline 577143 & 149.887635 & 2.175504 & $0.78 \pm 0.0$ & 0.0 & 0.0 & 0.11 & 10.7 & $15.76 \pm 1.443$ \\
\hline 580153 & 150.259862 & 2.181773 & $0.67 \pm 0.0$ & 0.01 & 0.02 & 0.05 & 10.2 & $5.035 \pm 1.073$ \\
\hline 581920 & 150.23722 & 2.183978 & $0.7 \pm 0.0$ & 0.08 & 0.09 & 0.01 & 10.2 & $17.91 \pm 1.338$ \\
\hline 584271 & 149.729701 & 2.186421 & $0.59 \pm 0.0$ & 0.0 & 0.0 & 0.1 & 10.6 & $16.49 \pm 0.88$ \\
\hline 586243 & 150.626379 & 2.187925 & $0.6 \pm 0.0$ & 0.01 & 0.01 & 0.08 & 10.0 & $5.475 \pm 1.482$ \\
\hline 586666 & 149.68814 & 2.191839 & $0.89 \pm 0.04$ & 0.01 & 0.01 & -0.11 & 11.0 & $146.4 \pm 13.37$ \\
\hline 586799 & 150.024476 & 2.190772 & $0.76 \pm 0.0$ & 0.01 & 0.02 & 0.08 & 10.4 & $9.945 \pm 1.957$ \\
\hline 587164 & 149.605886 & 2.190462 & $0.54 \pm 0.0$ & 0.06 & 0.07 & 0.02 & 10.8 & $13.38 \pm 0.944$ \\
\hline 587556 & 150.211575 & 2.191062 & $0.87 \pm 0.0$ & 0.01 & 0.01 & 0.07 & 10.9 & $25.36 \pm 0.477$ \\
\hline 588922 & 149.682152 & 2.193682 & $0.73 \pm 0.0$ & 0.07 & 0.07 & -0.02 & 10.4 & $23.22 \pm 0.597$ \\
\hline 601470 & 150.719972 & 2.211767 & $0.89 \pm 0.03$ & 0.03 & 0.03 & 0.06 & 10.5 & $34.8 \pm 5.655$ \\
\hline 606235 & 150.508123 & 2.219968 & $0.82 \pm 0.0$ & 0.01 & 0.01 & 0.03 & 10.2 & $48.24 \pm 0.716$ \\
\hline 607625 & 150.033442 & 2.221992 & $0.89 \pm 0.03$ & 0.05 & 0.06 & 0.0 & 10.3 & $14.44 \pm 1.223$ \\
\hline 608991 & 150.478377 & 2.22183 & $0.83 \pm 0.0$ & 0.03 & 0.03 & -0.0 & 10.5 & $45.74 \pm 2.175$ \\
\hline 609835 & 150.678868 & 2.225219 & $0.77 \pm 0.01$ & 0.0 & 0.0 & 0.04 & 10.2 & $59.46 \pm 2.104$ \\
\hline 619015 & 150.10376 & 2.237664 & $0.8 \pm 0.0$ & 0.02 & 0.03 & 0.08 & 10.7 & $100.7 \pm 1.634$ \\
\hline 620032 & 150.626231 & 2.240874 & $0.66 \pm 0.0$ & 0.04 & 0.05 & 0.06 & 10.0 & $9.124 \pm 0.871$ \\
\hline 622611 & 149.664939 & 2.241806 & $0.89 \pm 0.0$ & 0.01 & 0.01 & 0.06 & 11.3 & $12.98 \pm 3.813$ \\
\hline
\end{tabular}


Table A.1. continued.

\begin{tabular}{|c|c|c|c|c|c|c|c|c|}
\hline $\begin{array}{l}\text { ID } \\
(1)\end{array}$ & $\begin{array}{l}\text { RA } \\
(2)\end{array}$ & $\begin{array}{l}\text { Dec } \\
(3)\end{array}$ & $\begin{array}{l}\text { Redshift } \\
(4)\end{array}$ & $\begin{array}{l}c^{\prime} \\
(5)\end{array}$ & $\begin{array}{l}\text { Clumpiness } \\
(6)\end{array}$ & $\begin{array}{l}\text { Mergerness } \\
(7)\end{array}$ & $\begin{array}{l}M_{*}\left[M_{\odot}\right] \\
(8)\end{array}$ & $\begin{array}{l}\mathrm{SFR}_{\mathrm{IR}}\left[M_{\odot} \mathrm{yr}^{-1}\right] \\
(9)\end{array}$ \\
\hline 624991 & 149.9687 & 2.247664 & $0.85 \pm 0.0$ & 0.02 & 0.02 & 0.06 & 10.0 & $17.43 \pm 1.755$ \\
\hline 625435 & 149.538265 & 2.248029 & $0.84 \pm 0.0$ & 0.01 & 0.01 & 0.08 & 10.0 & $26.65 \pm 1.369$ \\
\hline 629919 & 150.477167 & 2.252186 & $0.84 \pm 0.0$ & 0.0 & 0.0 & 0.09 & 11.3 & $137.9 \pm 9.384$ \\
\hline 638793 & 149.529624 & 2.266751 & $0.58 \pm 0.0$ & 0.06 & 0.07 & -0.02 & 10.3 & $23.98 \pm 1.356$ \\
\hline 650054 & 150.400428 & 2.286241 & $0.83 \pm 0.16$ & 0.05 & 0.06 & 0.09 & 10.0 & $20.26 \pm 6.12$ \\
\hline 654259 & 149.872198 & 2.289675 & $0.7 \pm 0.0$ & 0.0 & 0.0 & 0.08 & 10.8 & $103.4 \pm 1.199$ \\
\hline 666872 & 150.537907 & 2.310505 & $0.84 \pm 0.0$ & 0.0 & 0.01 & 0.07 & 10.6 & $21.74 \pm 3.212$ \\
\hline 668065 & 149.937063 & 2.312279 & $0.52 \pm 0.0$ & 0.05 & 0.05 & 0.02 & 10.2 & $7.974 \pm 1.205$ \\
\hline 668738 & 150.210203 & 2.31168 & $0.75 \pm 0.0$ & 0.07 & 0.09 & -0.04 & 10.8 & $158.9 \pm 1.188$ \\
\hline 668769 & 149.67653 & 2.313653 & $0.69 \pm 0.0$ & 0.02 & 0.02 & 0.1 & 10.9 & $41.91 \pm 0.512$ \\
\hline 695086 & 150.05225 & 2.351018 & $0.72 \pm 0.13$ & 0.01 & 0.01 & 0.07 & 10.0 & $2.633 \pm 1.312$ \\
\hline 706850 & 149.931017 & 2.370359 & $0.85 \pm 0.03$ & 0.15 & 0.16 & -0.11 & 10.3 & $38.69 \pm 1.226$ \\
\hline 708427 & 149.578884 & 2.370704 & $0.75 \pm 0.0$ & 0.06 & 0.07 & 0.02 & 10.6 & $70.09 \pm 0.971$ \\
\hline 711307 & 150.53029 & 2.373861 & $0.66 \pm 0.0$ & 0.03 & 0.05 & 0.05 & 10.8 & $102.0 \pm 1.356$ \\
\hline 720848 & 150.261788 & 2.391079 & $0.8 \pm 0.0$ & 0.01 & 0.01 & -0.01 & 10.1 & $12.21 \pm 1.839$ \\
\hline 721398 & 150.670506 & 2.391459 & $0.61 \pm 0.0$ & 0.08 & 0.08 & 0.01 & 10.3 & $14.31 \pm 1.796$ \\
\hline 724796 & 150.597688 & 2.396014 & $0.58 \pm 0.0$ & 0.04 & 0.04 & 0.05 & 10.4 & $19.33 \pm 0.723$ \\
\hline 739926 & 150.470841 & 2.419552 & $0.55 \pm 0.0$ & 0.0 & 0.0 & 0.11 & 10.6 & $4.908 \pm 1.447$ \\
\hline 742281 & 150.641614 & 2.423434 & $0.73 \pm 0.0$ & 0.0 & 0.0 & 0.12 & 11.0 & $112.7 \pm 0.43$ \\
\hline 743322 & 149.657291 & 2.425131 & $0.57 \pm 0.0$ & 0.1 & 0.12 & 0.13 & 10.6 & $9.331 \pm 0.836$ \\
\hline 749126 & 150.427092 & 2.431647 & $0.88 \pm 0.0$ & 0.03 & 0.04 & 0.01 & 10.4 & $71.11 \pm 0.756$ \\
\hline 753450 & 150.327658 & 2.44088 & $0.52 \pm 0.0$ & 0.02 & 0.02 & 0.05 & 10.2 & $5.353 \pm 0.727$ \\
\hline 764862 & 150.189794 & 2.45758 & $0.51 \pm 0.0$ & 0.08 & 0.09 & -0.09 & 10.1 & $6.139 \pm 0.292$ \\
\hline 773897 & 149.833747 & 2.481315 & $0.84 \pm 0.0$ & 0.12 & 0.14 & 0.0 & 10.2 & $40.46 \pm 1.969$ \\
\hline 777034 & 150.150252 & 2.475166 & $0.69 \pm 0.0$ & 0.06 & 0.08 & -0.05 & 10.8 & $211.7 \pm 0.764$ \\
\hline 778756 & 150.548939 & 2.480459 & $0.8 \pm 0.0$ & 0.05 & 0.06 & -0.05 & 10.5 & $41.11 \pm 1.792$ \\
\hline 780365 & 150.398266 & 2.480753 & $0.85 \pm 0.0$ & 0.0 & 0.0 & 0.02 & 10.2 & $8.178 \pm 3.15$ \\
\hline 783499 & 150.506268 & 2.486894 & $0.62 \pm 0.0$ & 0.0 & 0.0 & 0.11 & 10.3 & $10.15 \pm 0.465$ \\
\hline 783743 & 149.93968 & 2.488034 & $0.72 \pm 0.15$ & 0.0 & 0.0 & 0.09 & 10.5 & $10.73 \pm 3.42$ \\
\hline 789914 & 149.979212 & 2.498304 & $0.88 \pm 0.0$ & 0.0 & 0.0 & 0.08 & 10.1 & $9.062 \pm 1.257$ \\
\hline 790685 & 150.370657 & 2.498115 & $0.82 \pm 0.0$ & 0.09 & 0.09 & 0.02 & 10.8 & $44.95 \pm 0.6$ \\
\hline 794024 & 150.055643 & 2.504436 & $0.7 \pm 0.0$ & 0.07 & 0.1 & 0.04 & 10.3 & $50.4 \pm 0.998$ \\
\hline 811857 & 149.913557 & 2.530162 & $0.69 \pm 0.0$ & 0.02 & 0.02 & 0.11 & 10.6 & $7.52 \pm 1.842$ \\
\hline 819000 & 149.457523 & 2.539333 & $0.83 \pm 0.02$ & 0.05 & 0.05 & 0.02 & 10.3 & $58.92 \pm 2.595$ \\
\hline 824508 & 149.862472 & 2.548484 & $0.74 \pm 0.0$ & 0.01 & 0.01 & 0.06 & 11.3 & $14.81 \pm 0.813$ \\
\hline 830418 & 149.562546 & 2.557087 & $0.76 \pm 0.03$ & 0.0 & 0.0 & 0.03 & 10.7 & $39.12 \pm 4.827$ \\
\hline 834449 & 150.1201 & 2.561479 & $0.5 \pm 0.0$ & 0.04 & 0.05 & 0.06 & 10.6 & $14.31 \pm 0.228$ \\
\hline 837890 & 150.054801 & 2.569459 & $0.75 \pm 0.0$ & 0.03 & 0.03 & 0.13 & 10.5 & $156.8 \pm 1.615$ \\
\hline 838188 & 149.77795 & 2.567252 & $0.72 \pm 0.0$ & 0.0 & 0.0 & 0.07 & 10.3 & $10.45 \pm 0.524$ \\
\hline 840232 & 149.716601 & 2.572475 & $0.9 \pm 0.01$ & 0.04 & 0.05 & 0.09 & 10.7 & $117.4 \pm 4.215$ \\
\hline 842149 & 150.342316 & 2.575943 & $0.75 \pm 0.0$ & 0.0 & 0.0 & -0.03 & 10.2 & $54.86 \pm 0.529$ \\
\hline 842173 & 149.733742 & 2.574995 & $0.71 \pm 0.0$ & 0.01 & 0.01 & 0.07 & 10.6 & $93.81 \pm 1.129$ \\
\hline 844990 & 149.926835 & 2.580581 & $0.7 \pm 0.0$ & 0.04 & 0.05 & 0.1 & 10.2 & $14.41 \pm 1.532$ \\
\hline 848785 & 150.42295 & 2.583241 & $0.81 \pm 0.0$ & 0.12 & 0.12 & -0.09 & 10.1 & $83.81 \pm 1.125$ \\
\hline 849397 & 149.976994 & 2.586831 & $0.73 \pm 0.0$ & 0.09 & 0.12 & 0.01 & 10.3 & $13.25 \pm 1.373$ \\
\hline 853769 & 150.33873 & 2.593234 & $0.85 \pm 0.0$ & 0.0 & 0.0 & 0.12 & 10.9 & $154.6 \pm 2.136$ \\
\hline 860071 & 150.27635 & 2.603899 & $0.7 \pm 0.0$ & 0.01 & 0.01 & -0.08 & 10.1 & $7.279 \pm 2.386$ \\
\hline 864706 & 150.531072 & 2.610881 & $0.79 \pm 0.0$ & 0.05 & 0.07 & 0.1 & 10.6 & $23.57 \pm 1.955$ \\
\hline 865896 & 150.054261 & 2.612264 & $0.74 \pm 0.0$ & 0.0 & 0.0 & -0.09 & 10.0 & $8.219 \pm 0.209$ \\
\hline 866054 & 149.811584 & 2.610245 & $0.54 \pm 0.0$ & 0.04 & 0.05 & 0.09 & 10.7 & $14.02 \pm 0.637$ \\
\hline 876155 & 150.564918 & 2.625331 & $0.65 \pm 0.02$ & 0.06 & 0.06 & 0.08 & 10.7 & $24.88 \pm 1.983$ \\
\hline 878476 & 150.752013 & 2.62904 & $0.84 \pm 0.11$ & 0.03 & 0.05 & 0.0 & 10.4 & $18.84 \pm 6.223$ \\
\hline 878551 & 150.046263 & 2.630447 & $0.76 \pm 0.03$ & 0.04 & 0.04 & 0.04 & 10.1 & $38.72 \pm 4.129$ \\
\hline 880787 & 149.550391 & 2.632955 & $0.81 \pm 0.06$ & 0.01 & 0.01 & 0.07 & 10.9 & $23.8 \pm 3.235$ \\
\hline 880925 & 149.937367 & 2.634136 & $0.71 \pm 0.03$ & 0.03 & 0.04 & 0.05 & 10.2 & $11.79 \pm 1.779$ \\
\hline 886498 & 149.513205 & 2.643508 & $0.64 \pm 0.0$ & 0.05 & 0.06 & +0.0 & 10.3 & $11.81 \pm 0.759$ \\
\hline 887351 & 150.04403 & 2.643885 & $0.7 \pm 0.0$ & 0.03 & 0.03 & -0.07 & 10.6 & $86.33 \pm 0.814$ \\
\hline
\end{tabular}


Table A.1. continued.

\begin{tabular}{lllllllll}
\hline \hline $\begin{array}{l}\text { ID } \\
(1)\end{array}$ & RA & Dec & $\begin{array}{l}\text { Redshift } \\
(3)\end{array}$ & $\begin{array}{l}c^{\prime} \\
(4)\end{array}$ & $\begin{array}{l}\text { Clumpiness } \\
(6)\end{array}$ & $\begin{array}{l}\text { Mergerness } \\
(7)\end{array}$ & $\begin{array}{l}M_{*}\left[M_{\odot}\right] \\
(8)\end{array}$ & $\begin{array}{l}\mathrm{SFR}_{\mathrm{IR}}\left[M_{\odot} \mathrm{yr}^{-1}\right] \\
(9)\end{array}$ \\
\hline 893857 & 150.159952 & 2.654341 & $0.85 \pm 0.0$ & 0.09 & 0.11 & -0.01 & 11.1 & $217.3 \pm 2.864$ \\
894779 & 150.427097 & 2.656439 & $0.55 \pm 0.0$ & 0.05 & 0.07 & -0.0 & 10.0 & $61.12 \pm 0.869$ \\
901597 & 150.709924 & 2.667676 & $0.7 \pm 0.08$ & 0.04 & 0.05 & 0.06 & 10.1 & $44.59 \pm 9.812$ \\
902885 & 150.136763 & 2.669235 & $0.7 \pm 0.0$ & 0.04 & 0.05 & 0.04 & 10.0 & $12.35 \pm 0.37$ \\
909617 & 149.970409 & 2.677765 & $0.69 \pm 0.0$ & 0.02 & 0.03 & 0.04 & 10.8 & $22.88 \pm 1.869$ \\
911723 & 149.681335 & 2.681084 & $0.58 \pm 0.0$ & 0.0 & 0.0 & 0.0 & 10.9 & $82.03 \pm 0.502$ \\
912969 & 150.589787 & 2.684536 & $0.74 \pm 0.0$ & 0.1 & 0.1 & -0.01 & 10.7 & $44.6 \pm 2.707$ \\
915913 & 150.557165 & 2.688555 & $0.88 \pm 0.0$ & 0.02 & 0.02 & 0.03 & 10.1 & $20.01 \pm 0.293$ \\
917600 & 150.029291 & 2.690141 & $0.79 \pm 0.09$ & 0.01 & 0.01 & 0.04 & 11.1 & $23.59 \pm 6.409$ \\
921254 & 149.910328 & 2.695579 & $0.86 \pm 0.14$ & 0.0 & 0.0 & 0.08 & 11.1 & $39.36 \pm 14.69$ \\
922401 & 150.035101 & 2.698678 & $0.65 \pm 0.0$ & 0.06 & 0.06 & -0.04 & 10.6 & $18.22 \pm 0.675$ \\
925981 & 150.433978 & 2.705011 & $0.88 \pm 0.0$ & 0.01 & 0.01 & 0.06 & 10.2 & $15.2 \pm 1.928$ \\
927031 & 149.716671 & 2.705636 & $0.88 \pm 0.0$ & 0.0 & 0.0 & 0.15 & 11.1 & $155.3 \pm 1.796$ \\
931390 & 150.586062 & 2.712214 & $0.66 \pm 0.0$ & 0.07 & 0.08 & 0.03 & 10.0 & $9.482 \pm 2.196$ \\
937909 & 149.79431 & 2.722162 & $0.9 \pm 0.02$ & 0.05 & 0.05 & 0.04 & 10.6 & $28.88 \pm 2.201$ \\
940851 & 150.539461 & 2.728218 & $0.66 \pm 0.0$ & 0.03 & 0.03 & 0.04 & 10.1 & $6.119 \pm 0.435$ \\
946233 & 150.227312 & 2.737645 & $0.7 \pm 0.09$ & 0.0 & 0.0 & 0.07 & 10.6 & $29.97 \pm 8.641$ \\
948557 & 149.627616 & 2.739277 & $0.55 \pm 0.0$ & 0.11 & 0.11 & -0.06 & 10.1 & $25.99 \pm 0.689$ \\
952356 & 149.886951 & 2.747311 & $0.88 \pm 0.0$ & 0.02 & 0.02 & 0.07 & 10.6 & $109.3 \pm 1.016$ \\
960767 & 149.848315 & 2.760829 & $0.7 \pm 0.0$ & 0.1 & 0.11 & 0.03 & 10.4 & $78.83 \pm 1.575$ \\
964786 & 150.126928 & 2.7654 & $0.57 \pm 0.0$ & 0.0 & 0.0 & 0.05 & 10.6 & $56.32 \pm 0.274$ \\
970636 & 150.408721 & 2.777253 & $0.84 \pm 0.09$ & 0.01 & 0.01 & 0.08 & 10.9 & $114.8 \pm 26.57$ \\
978011 & 150.155692 & 2.787663 & $0.73 \pm 0.0$ & 0.0 & 0.01 & 0.0 & 10.4 & $18.89 \pm 0.559$ \\
981123 & 150.399371 & 2.794152 & $0.82 \pm 0.0$ & 0.01 & 0.01 & 0.04 & 10.6 & $170.4 \pm 3.618$ \\
\hline
\end{tabular}

